\title{
A CHILD'S VOCABULARY AND ITS DEVELOPMENT
}

By John R. Phisma, Ph. M., University of Chicago

A. Vocabulary

\section{OUTLINE}

I. Biographical

I. Parentage

2. Association

3. Training

4. Biological data

II. Method of securing data

I. Daily conversation for three weeks

2. Questions framed to bring out known words

3. Naming objects

4. Monologue conversations

5. In fourth year child aided directly

III. Detailed vocabulary at the end of the first, second, third,

IV. Tables fourth years $\quad \cdot \quad \cdot \quad \cdot \quad \cdot \quad \cdot \quad \cdot \quad \cdot \quad \cdot \quad 334$

I. Per cent of nouns, verbs, etc., if "E's" vocabulary

2. Comparative list of vocabularies

3. Summary and arerages

V. Color vocabulary . . . . . . . . 346

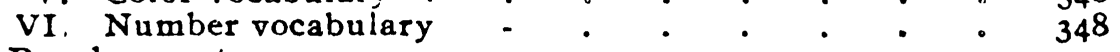

B. Development

I. Origin . . . • . • . . . .349

I. Theories advanced and corroborated from observation

II. Growth of " $\mathrm{E}$ "

r. Stages of growth
a. Reflex
b. Cry and gesture
c. Babbling
d. Imitation
e. Plateau
f. Expression

2. Method of growth

a. Reflex expression

b. Spontaneous emotional expression

c. Volitional expression

d. Imitation

e. Associative-reproduction

$f$. Invention

(r) From adults but get wrong form

(2) Proper words but wrong application

(3) Onomatopoetic sounds

(4) Onomatopoetic sounds applied to other objects

(5) Ejaculations

(6) Coustruction from "lol" words

(7) Counbinations

(8) Formation of regular variants 
B. Development

II. Growth

3. Systematic classification of progress made in different parts of speech

a. Statement

III. Pronunciation

I. Indistinct articulation

a. Undeveloped speech organs

b. Imperfect perception

c. Lack of memory

d. Imperfect example

e. Difficult combination

2. Development of correct pronunciation

IV. Sentence formation.$\quad \cdot \quad \cdot \quad \cdot \quad \cdot \quad \cdot \quad \cdot 364$

$r$. Stages

2. Methods

V. Word-content

Conclusion

INTRODUCTION

The human race has made many inventions enalsling it more perfectly to adapt itself to its environment. The greatest of all is Language. Language has been the open sesame to progress. Written langtrage represents in a condensed form the experiences and aspirations of all races. The thought and mental development of a people are limited by the number of words in its vocabulary and expressive gestures at its command. Accuracy of thought is in turn dependent on inflections and shades of meaning. Biologists affirm that ontogeny repeats phylogeny; in other words, the individual recapitulates the race. Though this theory can be accepted only with considerable modifications, philologists have frequently in their search for the genesis of speech turned to the babbling and prattling of the infant, in spite of the assertion of Max Miiller that he "feared it useless to watch the first stammering of children." There is a striking analogy between the development of speech and general intelligence in the human race and the child. From careful study of the ontogeny of speech in the infant, hypotheses have been deduced regarding phylogenetic origin of language (Darwin, Romanes, Beckman, Chanberlain. and many others). While educational psychology treats the development of the child between the ages of six and sixteen (Judd), a systematic study of the child before this age is of the utmost value to scientific education as well as of interest to philologists (Hall).

Many children's vocabularies have been recorded, but nearly all of them are individual cases. Mrs. Hall (ro) and Mr. Mickens (20) alone have published records of any extended attempt to note the progress in word forming processes in the same child for consecutive periods. Unfortunately their work 
was interrupted when the children were seventeen months and twenty-seven months old, respectively. Prof. H. N. Gale (7) has also given us the result of a similar study covering the period between the second and third years of the child's life. Meumann (4r) states that this study should continue until the fifth or sixth year, as the later years throw much light on the former. This article will submit a few facts concerning a child's vocabulary and a brief analysis of its development from birth until four years of age. Systematized data are yet too meager for conclusions which are entirely trustworthy. When more similar material has been gathered some future scientist may formulate general principles which will guide the investigator in further research which will lead to a further discovery and unification of general laws that catr ultimately be turned to practical account by the educator. Facts of every kind in regard to development of intelligence in children, including their progress in language, are important, but in the earlier stages of the investigation the common and ordinary facts rather than the unusual and extraordinary are the most valuable (Kirkpatrick (15).

\section{A. Vocabulary}

I. Biographical. Elizabeth Allegra Pelsma was born July 4, 1905, at Nappanee, Ind. After living here one and onehalf years she was taken to Reno, Nev. Remaining there one year, she was returned to Indiana. Her second summer, and a few odds weeks at other times, were spent on a farm, at the home of her maternal grand-parents in Northern Michigan. The six months prior to her fourth birthday were spent in Chicago. She has, therefore, had an opportunity to familiarize herself with the vocabulary of both city and country, as well as different sections of the country.

Her father is a teacher. Her mother is a high school graduate. Her father's nationality is Dutch. Her mother's, French aud Scotch-Irish and German. "E"' has, in the main, inherited the physique and temperament of her father.

She has been living with her parents continually, excepting a few days while visiting alone with her aunt. Her associates have been mainly adults. She has had little opportunity to play with children. She did not attend kindergarten until after she was four years old; has gone to Sunday School only a few times, and has had no opportunity to have a "chum" of near her own age, except for a few months. She has had no special instructions; cannot read one word, manifested no desire to read up to her fourth birthday; and has been given information only when she has demanded it, excepting such instructions as every American child has a right to expect from 
his parents and usually receives. Her questions have been answered when they were asked for the purpose of information, but were decidedly discouraged when asked for the mere purpose of asking questions. Such colloquy as the following has always been discouraged: "What are you going to do with that stick?" "Put it in the stove." "What are you putting it in the stove for?" "To burn." "What do you want to burn it for?" "To make heat." "What do you want to make heat for?" etc.

" $\mathrm{E}$ " has been permitted to grow up and live as "naturally" as possible. She has been permitted to have her own way much of the time. Absolutely no attempt has been made for systematic training of her intellect or emotions, as advocated by some educators (Sanford Bell). She has been permitted to follow the example of Rousseau's "Emile" whenever and wherever possible. When she has asked the name of an object or a process, she has been informed and the process explained in such language as she was able to comprehend. This was not done with a view of increasing her vocabulary but to acquaint her concerning her environment, and encourage that innate curiosity, without which development would be impossible. Her environment has been made as congenial and varied as circumstances permitted, and was deemed practicable. As she is the only child in the family, she is compelled to play much alone. Her mother has been her constant companion day and night. Her association with her father and otber adults has been unusually limited. Her father has been with her less than the average American father is with his child. During the first four years of her life her father has been obliged to be absent from home about ten out of the forty-eight months, or about one-fifth of the time.

Biologically "E"' may be termed a perfect child. She has always been strong, robust, and healthy; is symmetrically formed and developed. She has been the personification of health excepting a slight attack of lung fever in the fall of 1907 and a few days of illness incident to childhood. Her weight and height is as follows :

$\begin{array}{lcccccc} & \text { At birth } & 6 \text { mo. } & \text { r yr. } & 2 \text { yrs. } & 3 \text { yrs. } & 4 \text { yrs. } \\ \text { Weight lbs. } & 9.2 & 17.1 & 23.8 & 28.1 & 31.5 & 33.2 \\ \text { Height in. } & 21.0 & 26.6 & 29.7 & 35.2 & 38.1 & 40.6\end{array}$

\section{Method OF Securing Data}

Though no attempt was made to increase " $E$ 's' vocabulary faster than natural contingencies demanded, a rigorous attempt, however, was made to secure as far as possible all the words at her command during the special period of observation. 
The ten words in her vocabulary when she was twelve months old were secured without much trouble. To procure the vocabulary at the end of the second, third, and fourth years, demanded more skill and new methods. Three weeks prior to her $2 \mathrm{~d}, 3 \mathrm{~d}$, and $4^{\text {th }}$ birthdays, recorcling began and was continued uninterruptedly until her natal day. The plans were as follows: A tablet containing over twenty-six pages was obtained, and each page lettered alphabetically. On each page vertical columns were drawn corresponding to the number of the parts of speech. In this tablet all words, as soon as secured, were written on the appropriate page and in the proper column. Pieces of blank paper were tacked on the wall in a number of rooms, and a pencil fastened near by. On them her mother jotted such words as were used by ' $E$ "' in the absence of her father. With this co-operation of her mother a great many words were secured which would otherwise have been missed.

In all five distinct methods were used to secure the data: (I) Daily conversations with the child during the three weeks prior to her birthday. Most of the words were secured in this way. The child was very talkative and with a little encouragement would talk about her experiences of the day and of her desires for the morrow. (2) When it was believed that she knew the name of an object, action, attribute, or relation, and the word had not appeared in the conversation mentioned in (I), questions were framed to bring out the desired word in her answer. For example: Believing that she knew the word stove-poker, she was asked, "What does papa use to stir the fre?" She said, "shovel." "No, something long and round." She answered "poker." "How did the horses get across the water when you were riding with grandpa?" "They walked." "Did they walk in the water and get their feet wet?" "No, they walked on the bridge." (3) The direct naming of an object or an action. She would be shown a familiar object and asked to name it. The question usually assumed this form, "What is this?" If she knew she would name it. This method has been objected to by a few writers on children's vocabularies but the writer sees no reason why it is not legitimate. The child has the name of the object in his vocabulary and the reason it is not used in his ordinary conversation is because there has been no occasion. (4) The child at three years of age was frequently overheard to carry on an imaginary conversation with her dolls or playmates. These words were readily recorded. (5) In her fourth year ' $E$ '" was old enough to comprehend the purpose of being daily watched and followed with tablet and pencil. In answer to her inquiry she was told that papa wanted to write down all the words she knew. She 
took considerable interest in the proceeding and seemed proud that she knew so many words. Frequently she would come up to her papa and say: "I know - , have you that written down?" "U- is one of my words ; write that down." She seemed to delight in this and was very painstaking in having all her words written down in that tablet. This direct aid was of great importance in securing her vocabulary. Miss Mateer (19) objects to this method for the reason that the child may become self-conscious and refuse to talk, etc. This might be true with some children, but not with "E," and it is very doubtful if there are very many children who would become too self-conscious to express themselves naturally, they might with strangers, but not with those whom they were well acquainted with. Miss Shinn (49) corroborates this view.

The following tables claim no originality as to arrangement. The plan of Holden (II) has been deemed the must complete and practicable of any yet published, with the exception of Mrs. Hall's ( 15 ). However, Mrs. Hall's method contains phases which was not thought advisable to emphasize in this paper.

Writers are far from agreeing as to what should be included in a vocabulary such as is here recorded. Some would include proper names with common nouns. (Whipple (32). Moore (2r), Mateer (19).) Others would include inflected forms of verbs and adjectives (Whipple (32), Tracy (5I)). Until an agreement is reached as to what words shall be included in a standard vocabulary, averages and comparative tables are of little value. In "E's" third year vocabulary, proper nouns constituted $7 \%$ of her nouns; and in her fourth year, $10 \%$.

The following principles were observed in recording this vocabulary. Proper nouns are excluded. Present tense only of verbs when present tense was used by the child. No participial forms whatever, nor other inflected endings or grammatical variants. Articles have been included with adjectival forms. Singular number of nouns used in all cases when both singular and plural forms were known; but where only the plural form had been used, that form was recorded. Mr. Whipple's contention that every form should be included (excepting plurals) is more psychological than grammatical. All agree that "tall" and "taller" are two distinct conceptions in the child's mind, but how about goose and geese, or dog and dogs? Mr. Whipple has excluded plurals on the same principle that he has included am and was-a psychological rather than a grammatical basis. This exclusion does not seem consistent nor is this principle of classification practicable. It is true that the child sees no philological connection between 
tall and taller at two years of age, but how can it be told just when he does? Neither has the child but one concept for dog and dogs. They, too, are "distinct acquisitions" as well as tall and taller.

The only digression found in the following vocabularies, from the principle of reducing the number of words to a minimum, is in including all forms of the pronouns. No apology is made for doing this. Nearly all writers have done so, besides they are comparatively few, constituting in the fourth year less than two per cent. of the total number of words. Again, grammatically, parts of speech are determined by the use of the word in the sentence. No word has any part of speech, intrinsically; only when it becomes part of speech, as when used in a sentence. Therefore, the words as recorded have been given a substantive, verbial, or adjectival classification based on the manner and form as they were used by the child. "My below lip hurts." "Won't you hatchet this?" Here "below" has an adjectival form and "hatchet" is used as a verb-and both have been so classified. Great deviations from the common usage have been italicized. Invented words and others not sanctioned by the ordinary dictionary have been. placed in quotation marks. Compound words have been freely inserted when they were so expressed by the child. Frequently it was difficult to determine whether a word should be classified as a compound noun or as an adjective and a noun. Example, gasoline-stove, gasoline-can, etc. The word gasoline might properly be called an adjective, and stove, cow, etc., used as separate nouns. Such expressions were usually classified as compound nouns unless the adjectival form was frequently applied to unrelated objects.

\section{Detailed Vocabularies}

Age I2 months.

B. Nouns-Baby.

D. Nouns-Dada (daddy), dog.

Adverbs-Down.

G. Verbs-Go.

I. Nouns-."I" (water. A few months later this was changed to

K. Nouns-Kitty.

M. Nouns-Mama, man.

U. Adverbs-Uppe (up).

None of the above words were joined to form what is usually called a grammatical sentence, though each expressed a complete thought. (See IV under B.) 


\section{Detailed Vocabularies}

Age 24 months.

A. Nouns-Alarm-clock, alligator, apple, apron, arm, ashes, aunt.

Verbs-Am, ask.

Adjectives-All, any.

Adverbs-Almost, along, after, away.

Conjunctions-And.

3. Nouns-Baby, back, bacon, ball, banana, bank, barn, barrel, basket, bath, beans, bed, belt, bird, block, bonnet, book, bookcase, bottle, box, bracelet, breac, breakfast, bridge, broom, biggy, butter, button.

Verbs-Bite, break, bring, brush, build, bump, burn, button.

Adjectives-Bad, big, blue, both.

Adverbs-Back, behind, beside, bye.

Interjections-Boo.

C. Nouns-Cabbage, cap, cape, calf, lcau, candy, carpet, cart, cat, catalogue, chair, chicken, cherries, church, clock, closet, clothes, coal, coat, cocoa, comb, cookies, cream, cord, cow, cupboard.

Verbs-Can, can't, came, clean, carry, catch, cough, count, cry, cut.

C. Adjectives-Clean.

Adverbs-Careful.

D. Nouns-Dad, daddy, dimple, dinner, dish, dog, dollie, door, drink.

Verbs-Darn, dip, do, dress, drink, drop.

Adjectives-Dirty, dry.

Adverbs-Down.

E. Nouns-Ear, "eats"' (raisins), egg, eye.

Verbs-Eat.

Adjectives-Empty.

F. Nouns-Face, fan, fence, finger, finger-nail, fire, floor, flour, flower, fly, foot, fork.

Verbs-Fan, feed, feel, fix, fly.

Adjectives-Fat, flat, funny.

G. Nouns-Gurden, gate, girl, glass, go-cart, grandma, grandpa, grass.

Verbs-Get, give, go.

Adjectives-Good, green.

H. Nouns-Hair, hammer, hand, handkerchief, handle, hat, heel, home, horse.

Verbs-Hurry, hurt.

Adjectives-Hard, heavy.

Adverbs-Here.

Pronouns-He, him.

Interjections-Hello.

I. Nouns-Ice, ink.

Verbs-Is, is n't, itch (scratched).

Adverbs-In.

Pronouns-I, it.

J. Nouns-Jelly, Jumper.

Verbs-Junip.

K. Nouns-Key, kiss, kitty, knife.

Verbs-Kick, kiss.

L. Nouns-Lamp, lap, leaf, leg, lemon, letter.

Verbs-Let, lift, look, lose.

Adjectives-Little, long.

M. Nouns-Mail, mamma, man, meat, medicine, milk, wilkmau, moon, mother, mouse, mouth, mud.

Verbs-Make, may. 
M. Pronouns-Me, mine, my.

N. Nouns-Nail, name, neck, needle, night, "nighty," nose. Adjectives-New, nice.

Adverbs-No, now.

O. Nouns-Onion, orange.

Verbs-Open.

Adjectives-Old, one, other.

Adverbs-Off, out, over.

Prepositions-Of, on, over.

Interjections-Oh, öö, ouch.

P. Nouns-Pail, pan, pancake, papa, paper, patch, pan, petticoat, pie, pig, pillow, pin, plate, pop-corn, postage-stamp, potatoes (padils), pump.

Verbs-Paint, pinch, pound, pull, push, put.

Adjectives-Pretty.

Q. Nouns-Quilt ( $t w i l t$ ).

Verbs-Quit (twit).

R. Nouns-Rain, rake, rest, ribbon, robe, rocking-chair, rolling-pin.

Verbs--Rake, ride, ring, roll.

Adjectives-Red.

S. Nouns-Salt, sand, saucer, saw, school, sewing-table, shirt, shoes, side, sister, sitting-room, "skeeter," skin, snow, soap, spoon, steps, stockings, stove, strap, straw, string, supper, swing.

Verbs-Saw, scratch, see, sew, sing, sit, sleep, smell, sneeze.

Adjectives-Sick, sleepy.

Interjections-Shoo.

T. Nouns-Table, table-cloth, tail, teeth, thing, thread, throat, tincup, titty, toast, toes, tongue, tooth-picks, top, town, train, tub. Verbs-Tack, take, talk, tell, tickle, tie, tip, try, turn.

Adjectives-That, the, this, tiny, tired, two.

Adverbs-There, then.

Prepositions-Through, to.

U. Nouns-Uninn-suit, up-stairs.

Verbs-Unbutton, "unlocken" (unlocked).

Adverbs-Up.

V. Nouns-Vessel (bettaw).

W. Nouns-Wash-board, watch, water (ä-ja), whip, wood

Verbs-Walk, wash, wear, wet, whip, wipe, wrap.

Adjectives-Wet, white.

Adverbs-When.

$\mathrm{X}$.

Prepositions-With.

Y. Adjectives-Yellow.

$Z$.

Prowouns-You.

Age 36 months.

A. Nouns-Alarm-clock, alligator, apple, apron, aral, ashes, aunt, automohile, ax.

Verbs-Am, are n't, ask.

Adjectives-Afraid, all, any.

Adrerbs-After, almost, along, away.

Conjunctions-And.

B. Nouns-Baby, back, bacon, ball, banana, bank, barrel, barn, basket, bath, beans, bed, bell, belt, berries, bib, bicycle, bird, bite, black-berries, blanket, black, blood, board, body, bone, bonnet, hook, book-case, bottle, bowl, box, boy, bracelet, braid, bread, breakfast, breakfast-food, brick, bridge, broom, brush, bucket, bug, buggy, bump, butter, button. 


\section{A CHILD'S VOCABULARY}

B. Verbs-Bake, bathe, bend, bite, bleed, blow, boil, bother, break, bring, brusb, build, bump, burn, burst, button, buy.

Adjectives-Bad, bare-foot, better, big, bitter, black, blue, both, brown, busy.

Adverbs-Back, before, behind, beside, bye.

Prepositions-By.

Interjections-Boo.

C. Nouns-Cabbage, cake, calf, can, candy, cap, cape, car, card, carpet, cart, cat, catalogue, chair, chalk, cherries, chest, chicken, cburch, churn, cinnamon, o'clock, closet, clothes, clothes-line, clothes-pin, clothes-rack, coal, coal-pail, coat, cocoa, coffee, cold-cream, collar, comb, comforter, cookies, corn, corner, corset, couch, cough, crackers, cream, crock, curtain, cuff, cuffbutton, cup, cupboard.

Verbs-Can, can't, care, carry, catch, chase, clean, climb, close, come, cough, count, cry, cut.

Adjectives-Clean, cold, cute.

Adverbs-Carefully.

D. Nouns-Dad, daddy, darling, daughter, dimple, dinner, dipper, dirt, dog, dollie, door, dress, drink, drum.

D. Verbs-Darn, die, dig, dip, do, don't, dress, drink, drive, drop, drum, dust.

Adjectives-Dark, dirty, dry.

Adverbs-Down.

E. Nouns-Ear, eating-apron, egg, elbow, end, euvelope, eye, eyebrows.

Verbs-Eat, excuse.

Adjectives-Empty.

F. Nouns-Face, fan, feather, feather-duster, fence, finger, fingernail, finger-stall, fire, fish, flag, floor, flour, flower, fly, foot, fork.

Verbs-Fall, fan, feed, feel, find, fix.

Adjectives-Fat, first, flat, four, fresh, full, funny.

Adverbs-Far, fast.

G. Nouns-Garden, gate, girl, glass, glasses (eye-glasses), go-cart, grandma, grandpa, grapes, grass, grater, ground.

Verbs-Get, give, go, grow, grunt, guess.

Adjectives-good, gray, green.

Adverbs-Good-night.

H. Nouns-Hair, hair-pin, hammer, hamper, hand, handkerchief, handle, hat, hatchet, hay, head, heel, hiccough, hobby-horse, hoe, home, horse, house.

Verbs-Have, hear, hit, hold, hug, hurry, hurry, hurt, hunt.

Adjectives-Hard, heavy, hot, hungry.

Adverbs-Here.

Pronouns-He, her, him, his.

Interjections - Ho, hough.

I. Nouns-Ice, ice-cream, ice-man, ice-wagon, ink, iron, itch.

Verbs-Iron, is, is n't.

Adverbs-Inside.

Prepositions-In.

Pronouns-I, it.

J. Nouns-Jelly, juice, jumper.

Verbs-Jump.

K. Nouns-Kettle, key, kiss, kitchen, kitty, knee, knife, knot.

Verbs-Kick, kiss, know.

L. Nouns-Lace, lady, lamp, lamp-chimuey, lap, lard, leaf, leg, lemon, lemonade, letter, light, line, lip, looking-glass.

Verbs-I,et, lift, light, like, "locken" (locked), look.

Adjectives-Last, light, little, long. 
M. Nouns-Mail, mail-box, mail-man, mama, man, meat, medicine' milk, milk-man, mirror, monkey, moon, mouse, mouth, mouth organ, mother, mud, mustache.

Verbs-Make, may, mix.

Pronouns-Me, mine, my.

N. Nouns-Nail, name, neck, necktie, needle, night, night-drawers, "nighty", noodles, nose.

Verbs-Nail.

Adjectives-New, nice.

Adverbs-Never, no, now.

O. Nouns-Oil, oil-can, oil-cloth, onion, orange, oven.

Verbs-Open.

Adjectives-Old, one, other.

Adverbs-Off, on, out, over.

Prepositions-Of, on, over.

Interjections-Oo, ob, oh-my, ouch.

P. Nouns-Pail, paint, paint-brush, pan, pan-cakes, pantry, pants, papa, paper, paste-board, patch, pattern, peanuts, pears, pen, pencil, penny, people, pepper, petticoat, piano, picture, pie,

P. Nouns- piece, pig, pig-pen, pillow, pin, pin-tray, pitcher, plant, plate, pocket, pocket-book, porch, post, postage-stamp, postal. card, powder, potatoes, pump, pusher, pussy.

Verts-Paddle, paint, pat, pick, pin, pinch, plant, play, pound, pull, pump, push, put.

Adjectives-Pink, pretty.

Q. Nouns-Quilt.

Verbs-Quit.

Adverbs-Quiet.

R. Nouns-Rack, radish, rain, raisin, rake, raspberry, ribbon, ring, robe, rocking-chair, rolling-pin, rope, rubbers, rug.

Verbs-Rain, rake, rest, ride, roll, run.

Adjectives-Red, right.

S. Nouns-Sack, safety-pin, salt, sand, sandless, sand-pile, saucer, saw, school, scissors, screw, sewing, sewing-machine, sewing-machine-drawer, sewing-table, sheet, shirt, shirt-waist, shelf, shoe, shoe-string, shovel, side, side-walk, sister, sitting-room, skeeter, skillet, skin, skirt, sleeve, slipper, snow, soap, socks, soup, spade, splinter, spoon, star, starch, steps, stick, stockings, stone, stool, strap, stove, straw, street, street-car, string, sugar, supper, supporters, swing.

Verbs-Say, see, sew, scare, scratch, scour, shove, shut, sing, sit, sleep, sneeze, snow, smell, sort, stay, swing.

Adjectices-Sick, sleepy, some, sore, sorry, sour, sweet.

Interjections-Shoo.

T. Nouns-Table, table-cloth, tack, toil, tears, Teddy-bear, teeth, telescope, thing, thread, throat, thumb, tie, time, tin-cup, titty, toast, toes, toilet, tomatoes, tongue, tooth-picks, top, towl, town, toy, train. trunk, tub.

Verbs-Tack, talk, tear, tell, thank, throw, tickle, tie, try, turn.

Adjectives-That, the, this, three, tight, tiny, tired, two.

Adverbs-There, through, too.

Prepositions-Through, to.

Pronouns-Them, they.

$U$. Nouns-Umbrella, union-suits.

Verbs-Unbutton "unlocken."

Adverbs-Up, up-stairs.

Prepositions-Up.

Pronouns-Us.

V. Nouns-Vaseline, vessel, vinegar.

Adverbs-Very. 
W. Nouns-Walk, watch, water, wash-board, wash-boiler, wash-tub, waist, way, wheel, wheel-barrow, whip, whistle, window, wood, writing-desk.

Verbs-Wait, walk, want, wash, watch, wear, wet, whip, wipe, wrap, write.

Adjectives-Well, white.

Adverbs-When.

X.

Prepositions-With.

Y. Adjective6-Yellow.

Z.

Pronouns-Your, yours.

Age 48 months.

A. Nouns - Afternoon, alligator, ankle, anybody, apple, apricot, apron, arm, aunt, automobile.

Verbs-Am, aren't, ask.

Adjectives-A, afraid, all, an, another, any, around, awful.

A. Adverbs-Afterwhile, again, alike, along, almost, alone, alright, anywhere, apart, around, away.

Prepositions-Across, after, at, around.

Conjunctions-And.

Interjections-Ah.

B. Nouns-Baa, baby; back, bacon, ball, bologna, banana, band, barn, barn-yard, barrel, basket, bath, bath-room, beads, beans, bed, bed-room, beetle, bench, bell, belt, belt-buckle, berry, berryboxes, bib, bicycle, bird, birthday, bite, biter, black-berries, blanket, block, blood, blueing, boat, boat-rider, boards, body, boiler, bone, bonnet, book, book-case, bottle, bottom, bouncingball, box, boy, braid, bread, bread-dough, bread-pan, breakfast, breakfast-food, brick, bridge, broom, brother, brusk, bucket, bug, buggy, bump, butter, butter-bowl, butterfly, button, button-hole, button-hook.

Verbs-Bake, bark, bathe, beat, behave, bend, bite, blow, boil, bother, born, break, bring, brush, build, burn, bust, button, buy.

Adjectives-Baby, bad, barefoot, better, big, bitter, black, blue, blue-eyed, both, bouncing, brown, bum, busy.

Adverbs-Back, backward, before, behind, besides, better, bye.

Prepositions-Behind, beside, by.

Conjunctions-But.

Interjections-Baa, bowwow.

C. Nouns-Cab, cabbage, cage, cake, calf, can, candy, cap, car, cart, carrot, cat, catalogue, catchup, card, cards, carpet, caster-oil, chain, chair, cheek, cheese, cherries, chest, chewing-gum, chick, chicken, shildren, chimney, chin, chocolate, Christmas, church, churn, ceiling, cellar, cemetery, cigar-box, cinnamon, class, cloak, clock, closet, cloth, clotkes, clothes-line, clothespin, cloud, coal, coal-oil, coat, cock-a-doodle-doo, cocoa, coffee, "coffee-churn," cold, collar, comb, comforter, conductor, cooky, cork, corn, corset, cough, cough-syrup, cousin, cover, cow, crack, cracker, cracker-jack, crayons, cream, cricket, crock, crust, cuff, cuff-button, cup, cup-board, "cup-egg," curtain.

Verbs-Call, can, can't, care, carry, catch, chase, churn, clean, clime, close, come, cough, count, cover, crack, crawl, cry, cut.

Adjectives-Careful, chocolate, chrome-yellow, clean, clear, close, cold, cute.

Adverbs-Clear, careful.

D. Nouns-Dad, daddy, dandelion, darning-cotton, daughter, dear, dentist, dime, dimple, dining-room, "dinky," dinner, dipper, 
D. Nouns-Dirt, dish, dish-pan, dish-towel, doctor, dog, doggie, donkey, door, drawer, dream, dress, dresser, dressing, drink, drop, drum, duck, dust.

Verbs-Darn, dip, do, don't, draw, drink, dress, drop, dry, dust.

Adjectives-Dandy, dark, dark-blue, dark-brown, dead, dirty done, dusty.

Adverbs-Down, down-stairs.

E. Nouns-Ear, eating-apron, edge, egg, egg-beater, elbow, elevator: elevated, end, engine, envelope, eraser, eveniug, everybody, everything, eye, eye-brows, eye-winker.

Verbs-Eat, empty, excuse.

Adjectives-Each, early, empty, every.

Adverbs-Early, easily, either, else.

F. Nouns-Face, face-powder, fan, feather, feather-duster, fence finger, finger-nail, fire, fire-cracker, field, fish, flag, flat, floor flour, flour-sifter, flower, fly, food, foot, forehead, fork, friend frog, front, fudge, funeral.

F. Verbs-Fall, fan, faster, feed, feel, figure, find, finish, fix, $\mathrm{fly}_{\text {, }}$ float, forget.

Adjectives-Fat, first, five, flat, four, four-year-old, fresh, funny, full.

Adverbs-Far, fast, first, forth.

Preposition-For.

G. Nouns-Garden, gasoline, gasoline-stove, gate, girl, glass, glasses, glue, gloves, go-cart, gold-fish, goose, grandua, grandpa, grapes grater, groceries, ground.

Verbs-Get, giggle, give, go, grow, grunt, guess.

Adjectives-Gasoline, good, graham, gray, great, greasy, green

Adverbs-Good, good-bye, good-night, great.

Interjections-Goodness' sake.

H. Nouns-Hair, hair-pin, hair-rat, hair-ribbon, half, hall, hall-way. hand, handkerchief, handle, hammer, hot, hatchet, hat-pin, head, hearse, heart, heel, hide-and-go-seek, hiccough, hickory. nut, high-chair, hive, hobby-horse, hoe, hole, home, honey, hood, hooks, horns, horsie, hubby, hydrant.

Verbs-Ha'n't, hatchet, have, hear, help, hide, hike, hit, hold, holler, hug, hunt, hurt, hurry, hustle.

Adjectives-Hard, heavy, high, hot, bungry.

Adverbs-Hardly, hear, how.

Pronouns-He, her, hers, him, his.

Interjections-Hello, hey, ho.

I. Nouns-Ice, ice-cream, ice-man, ice-wagon, ink, ink-bottle, irou, ironing-board, itch.

Verbs-Iron, is, isn't, itch.

Adverbs-Inside.

Pronouns-I, it.

Prepositions-In, into, inside.

Conjunctions-If.

J. Nouns-Jacket, jelly, joke, juice, jumper, jumping-jack.

Verbs-Jerk, jump.

Adverbs-Just.

K. Nouns-Kettle, key, kick, kids, kinkling, kiss, kitchen, kitchencabinet, kitty, knee, kuife.

Verbs-Kick, kill, kiss, knock, know.

Adverbs-Kinda.

L. Nouns-Lace, lady, lake, lamp, lamp-chimney, lamp-lighter, lap, lard, laundry-bag, lawn-mower, leaf, leg, lemon, lemouade, letter, letters, library light, lightning, line, lip, liver, load, loaf, lodge, lounge, lump, lunch. 
L. Verbs-Lay, left, let, lick, lift, light, like, listen, lock, look, loose, love, lye.

Adjectives-Last, left, light, light-brown, little, long, loose, loud, low.

Adverbs-Late, like, lot.

Interjections-Loo-loo.

M. Nouns-Magazine, mail, mail-box, mail-man, mamma, man, marble, mark, match, meat, meat-saw, medicine, milk, milk-bottle, milk-man, milk-toast, milk-wagon, minute, mirror, mistake, mitten, mole, money, monkey, moon, moon-shine, morning, mosquito, mother, motor-man, mouse, mouth, moving-pictureshow, mud, muff, muffler, mush, mustard.

Verbs-Make, march, mark, match, may, mean, mend, "mickeled" (mussed), might, milk, mind, miss, mix, move, must.

Adjectives-Mad, many, merry, middle, much.

Adverbs-More, most, much.

M. Pronouns-Me, mine, my, my-own, my-own-self, my-self.

N. Nouns-Nail, name, nap, napkin, neck, neck-tie, needle, newspaper, night, night-drawers, night-gown, "nighty." nobody, noise, noodles, nose, nothing, nurse, nuts.

Verbs-Nail, name, need, nurse.

Adjectives-Naughty, near, new, next, nice, no, none.

Adverbs-Nearly, never, no, now.

O. Nouns-Oil, oil-can, oil-cloth, olives, onions, orange, oven, oxen. Verbs-Oil, open, order.

Adjectives-Old, one, orange, other.

Adverbs-Off, on, once, open, out, outside, over.

Pronouns-Ownself.

Prepositions-Of, on, out, over.

Interjections-Oh, ouch.

P. Nouns-Package, packer, pad, page, pail, paint, paint-brush, pan, pan-cakes, pantry, pants, papa, paper, paraffin, parasol, parlor, parrot, part, patch, peach, peanuts, peas, pen, pencil, penny, people, pepper, peppermint, period, petticoat, piano, pickle, picture, picture-book, pie, piece, pig, piggie, pile, pills, pillow, pimple, pin, pineapple, pin-tray, pitcher, place, plant, plate, "plateegg," plum, pocket, pocket-book, poker, pop-corn, porch, postage-stamp, postal-card, posy, potato, potato-cakes, potatomasher, powder, prayer, price, prunes, pudding, pump, pumpkin, pusher, pussy.

Verbs-Paint, paddle, part, paste, pat, patch, pay, pick, pile, pin, pinch, plant, play, poke, pound, pull, pamp, punch, push, put. Adjectives-Plaid, pink, pretty, purple.

Adverbs-Perhaps, please.

Q. Nouns-Question; question-mark, quilt.

Verbs-Quit.

Adverbs-Quick, quiet.

Interjection-Quack.

R. Nouns-Rabbit, raddish, rag, rain, rain-bow, raisins, rake, range, rat, rat-trap, raveling, rest, ribbon, rice, ride, ring, robe, robin, rocking-chair, "rolabola," rolling-pin, rompers, roof, room, rooster, rope, rubbers, rug, ruler.

Verbs-Rain, raise, rake, read, remember, rest, ride, rock, roll, rub, run.

Adjectives-Ready, red, right, rough, round.

Adverbs-Kight, (a)round.

S. Nouns-Sack, safety-pin, salt, salt-shaker, salve, sand, sandals, sand-pile, saucer, sausage, saw, saw-dust, scales, scare, school, scissors, screen, screen-door, scouring-soap, screw, screw-eye, 
S. Nouns-screw-driver, seam, seat, seed, set, sewing, sewing-machine, sewing-machine-drawer, sewing-table, shadow, shave (lather), shawl, shears, sheep, sheet, shell, shirt, shirt-waist, shoe, shoestring, shoot, short-cake, shot-gun, shovel, side, side-walk, sink, sister, skates, skillet, skin, skirt, sky, slap, sled, sleeve, slipper, smoke, snow, soap, soap-dish, soap-suds, socks, something, soup, space, spade, spare-ribs, spinning-top, splinter, spool, spoon, stairs, stair-way, stand, star, starch, station, step, steps, stem, stick, stocking, stomach, stomach-ache, stone, stool, stopper, store, store-room, story, story-book, strap, strawberries, street, street-car, string, stuff, suds, sugar, sugar-bowl, sugarspoon, summer, sun, sun-shine, supper, supporters, sweet-heart, swing, syrup.

S. Verbs-Say, saw, scratch, see, send, set, shake, shall, shave, shine, shoot, shout, shove, shovel, show, shut, sing, sit, skate, slap, sleep, slide, slip, smell, smoke, sneeze, sort, sow, spank, speak, spell, spill, spin, spit, spoil, sprinkle, stand, starch, stay, stick, stir, stop, string, study, stumble, suck, swallow, sweat, swim, swing.

Adjectives-Sharp, short, sick, six, sleepy, slick, slow, snowy, soft, such, sweaty, sweet.

Adverbs-Some, sometimes, so, sore, sure.

Interjection-Shoo.

T. Nouns-Table, table-cloth, tack, tack-hammer, tail, talcum-powder, tape-line, tea, teacher, tears, tea-spoons, tea-towel, Teddybear, telephone, telescope, thimble, thing, thread, throat, thumb, thunder, ticket, ticket-puncher, tights, time, tin, tin-cup, tire, tissue-paper, titty, toast, to-day, tomatoes, to-morrow, toe, toenail, toilet, tongue, teeth, tooth-ache, tooth-brush, tooth-pick, top, towel, town, train, trap, trash, tree, trouble, trousers, trunk, tub, tuck, tumbler.

Verbs-Take, talk, taste, teach, tear, tease, telephone, tell, thank, throw, tickle, tie, touch, try, tumble, turn.

Adjectives-Tall, tan, that, the, these, thin, thirsty, this, three, tight, tiny, tired, toasted, to-morrow, two.

Adverbs-Then, there, through, till, to-day, together, to-morrow, too.

Pronouns-That, this, they, them.

Prepositions-Till, to.

Conjunctions-Than, then.

Interjections-There, toot-toot.

U. Nouns-Umbrella, uncle, union-suits.

Verbs-Unbutton, undress, unfasten, unlock, untie, use.

Adjectives-Ugly.

Adverbs-Up, upstairs.

Pronouns-Us.

Prepositions-Under.

V. Nouns-Vaseline, vessel, vest, vinegar.

Adverbs-Very.

W. Nouns-Wagon, waist, wall, wall-paper, walk, wart, wash-board, wash-boiler, wash-bowl, washing-day, wash-pan, wash-rag, washtub, watch, watch-chain, water, water-melon, water-pail, waterpitcher, wax, way, week, weenies, wheel, wheel-barrow, whip, whiskers, whopper, wig, wind, window, wings, "winkie," winter, wire, woman, wood, word, work, worker, wringer, wrist.

Verbs-Wait, wake, waked-up, walk, watch, water, want, wash, wear, wet, whip, whisper, whistle, wiggle, will, wipe, wire, wish, won't, wonder, work, would, wrap, wring, wrinkle, write.

Adjectires-Warm, well, wet, white, whole, wrinkled, writing, wrong. 
W. Adverbs-Way, what, when, where.

Pronouns-Who.

Prepositions-With.

$\mathrm{X}$.

Interjection-Whoa.

Y. Nouns-Yeast.

Adjectives-Yellow, yellow-ochre.

Adverbs-Yes, yesterday, yet.

Z.

Prououns-You, yours, yourselves.

Proper nouns are not included with the rest of the rocabulary.

During the first year no proper names were spoken. The first proper name attempted was her own name, Elizabeth. This was first pronounced "Isbes," next "Isbe.," "Isbeth,", "Lisbeth," and not until she was four years old was the name pronounced correctly.

During the three weeks preceding her second birthday she used the following words: Elizabeth, Net, Turner, Sis. -4 in all.

At her third birthday she used the following: Anna, Berry St., Claudie, Cloa, Clarence, College Ave., Mrs. Crowley, Dorothy, Earl, Effie, Ella, Floyd, Florence Hall, Mrs. Gardiner, Goshen, Greencastle, Harry, Herb, Mrs. Hill, John, Kermet, Lake City, Lena, Maude, Myrtle, Minnie, Pelsma, Mrs. Stephenson-28 in all.

At her fourth birthday the following proper names were used: Anna, Allegra, Bertha, Blanche, Bud, Chicago, Cloa, Carl, Claude, Mrs. Crawley; Dennis, Dorothy, Elizabeth, Ella, Emma, Eastern Star, Edward, Ethel, Floyd, Florence, Florence Hall, Friday, Fourth of July, George, Genevieve, Glen, God, Grace, Grand Rapids, Mrs. Gardiner, Greencastle, Hazel, Herb, Helen, Mrs. Hill, Indiana, Jackson Pa: $\therefore$, Jackson Ave., Jennie, Jesus, John, Katherine, Klass, Kermet, Kitty, Lake City, Laurence, Lincoln Park, Lord, Mary, May, Margaret, Masonic Lodge, Minnie, Maud, Monday, Mildred, Nellie, Noel, O'Conner, Raymond, Robinson, Rebecka Lodge, Saturday, Sunday, Mrs. Stepheuson, Santa Claus, Thursday, Tuesday, Wednesday, Mrs. White, White City, Washington, Washington Park-78 words in all.

"E") also knew all the letters of the alphabet except $Q, Y$ and $Z$. It was surprising how eagerly she sought to learn these arbitrary symbols. Attempts were made to teach her simple words, but she did not care to learn these, preferring the letters, and would insist continually that we tell her the name of a letter. She would spell out a word and then ask us to pronounce the word. She did not become interested in the words themselves until the last part of her third year. Technically speaking, the letters are the names of the symbols 
they represent, and grammatically may be called nouns. This would increase her total vocabulary, including proper nouns, and the twenty-three letters of the alphabet, to 1,379 words. This total still seems low when compared with Whipple's three-year-old boy who had I,77I, Jegi's two-yearold boy with I, 227, or Humphrey's two-year-old boy with I, I 2 I words. But it must be remembered that they had exceptionally bright children, and also included in their tables some grammatical variants, $i$. e., inflections as: go, going, gone; comparisons, as tall, taller, tallest, and all compounds.

\section{TABLES}

Table (I) shows a progressive summary which needs no comment.

Table (2) gives a detailed list of children's vocabularies up to four years of age which have been published. Seventy-two are included. Dr. Tracy $(5 \mathrm{I})$ summarized the vocabularies of about i 8 children. Prof. Whipple (32) adds about 18 to the list of Dr. Tracy. Table (2) gives the total numbers of words and parts of speech.

Table (3) presents a complete summary of twenty-six vocabularies classified by years, and sixty-eight by groups. Parts of speech and per cent. are given. Lists which were not complete in Table (2) have been exciuded. An attempt has been made to include all vocabularies which were not questionable, not merely the greatest. Yet we fear that the average is still very high, as most of the records are of precocious children.

Gale (8) states that the vocabulary of the average two-yearold child is 700 , and 1,400 at $21 / 2$ years. This is too high. Our table for 14 children at two years gives 518 words; and at three years only 1,209 . This is still too high for the average three-year-old, for our average at four years is only 1,149 . Insufficient data exist at present. We believe that when more data are accumulated that 'E's' vocabulary will be found not far from that of the average American child; for " $E$ " is in every way a child of only average ability. She was not like Jegi's child, who at two years of age had outgrown Mother Goose Rhymes! Such exceptional cases as the child of Dr. E. Gates ( 17 ), who at the age of 2 I months knew I I, 00O (?) words, are of little practical value to the educator. We need more data from normal children. Again, it will be found that 2,0,00 words is not far from the number of words at the command of the average six-year-old child-i.e., at the time he enters schoo!. 
PELSMA, JOHN R., A child's vocabulary and its development, Pedagogical Seminary, 17 (1910) p.328-369

A CHILD'S VOCABULARY

345

1. The following Table presents a progressive summary of " $E$ ' $s$ " vocabulary in number of words and in per cents.

\begin{tabular}{|c|c|c|c|c|c|c|c|}
\hline \multirow[b]{2}{*}{$\begin{array}{l}\text { Parts of speech } \\
\text { Nouns } \\
\text { Verbs } \\
\text { Adjectives } \\
\text { Adverbs } \\
\text { Pronouns } \\
\text { Prepositions } \\
\text { Conjunctions } \\
\text { Interjections }\end{array}$} & $\begin{array}{l}\text { First year } \\
\text { words } \%\end{array}$ & \multicolumn{2}{|c|}{$\begin{array}{l}\text { Second year } \\
\text { words } \%\end{array}$} & \multicolumn{2}{|c|}{$\begin{array}{l}\text { Third year } \\
\text { words } \%\end{array}$} & \multicolumn{2}{|c|}{$\begin{array}{l}\text { Fourth year } \\
\text { words } \%\end{array}$} \\
\hline & $\begin{array}{l}70 \\
10 \\
20\end{array}$ & $\begin{array}{r}212 \\
88 \\
37 \\
21 \\
8 \\
6 \\
1 \\
6\end{array}$ & $\begin{array}{r}56.0 \\
23.3 \\
9.9 \\
5.6 \\
2.7 \\
1.6 \\
.3 \\
1.6\end{array}$ & $\begin{array}{r}406 \\
147 \\
65 \\
31 \\
14 \\
9 \\
1 \\
8\end{array}$ & $\begin{array}{r}59.8 \\
21.4 \\
9.8 \\
4.6 \\
2.0 \\
1.2 \\
.1 \\
I .1\end{array}$ & $\begin{array}{r}730 \\
265 \\
135 \\
86 \\
23 \\
19 \\
5 \\
15\end{array}$ & $\begin{array}{r}56.6 \\
20.7 \\
10.6 \\
6.8 \\
1.9 \\
1.6 \\
.5 \\
1.3\end{array}$ \\
\hline Total & 100 & 379 & 100 & $68 I$ & 100 & 1,278 & 100 \\
\hline
\end{tabular}

2. Comparative Table

Classification into Parts of Speech

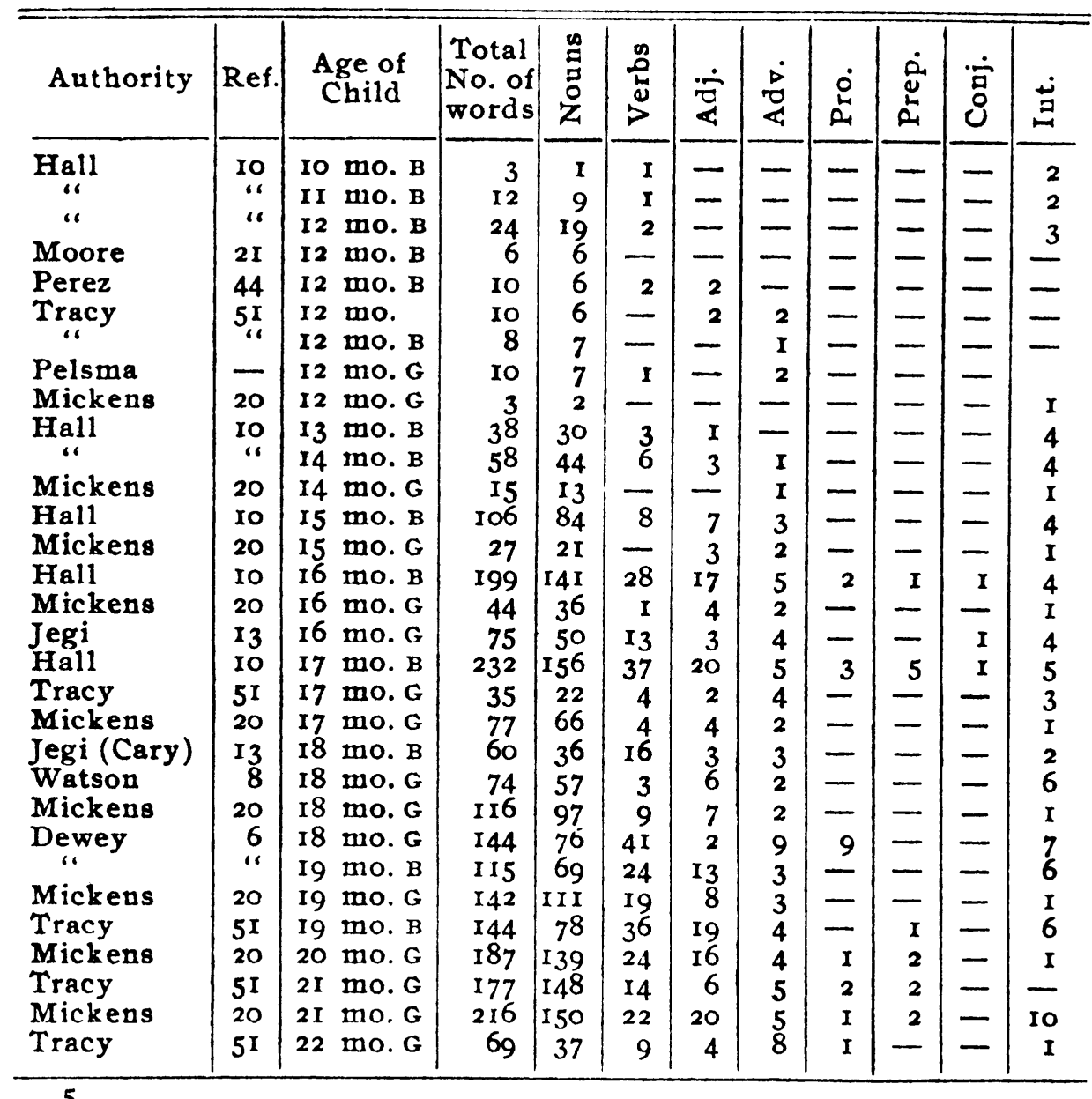


PELSMA, JOHN R., A child's vocabulary and its development, Pedagogical Seminary, 17 (1910) p.328-369

A CHILD'S VOCABULARY

2. Comparative TaBLF-Continued

\begin{tabular}{|c|c|c|c|c|c|c|c|c|c|c|c|c|c|}
\hline Anthority & Ref & & $\begin{array}{l}\text { Age o } \\
\text { Child }\end{array}$ & & $\begin{array}{l}\text { Total } \\
\text { No. of } \\
\text { words }\end{array}$ & z & $\frac{8}{5}$ & 它 & 娄 & 눈 & Pي & $\begin{array}{l}\dot{0} \\
\dot{0}\end{array}$ & $\stackrel{\leftrightarrow}{ٌ}$ \\
\hline Mickens & 20 & 22 & no. & & $28 I$ & 185 & 49 & 27 & II & 2 & 4 & I & 2 \\
\hline Tre & $5 I$ & 22 & mo. & & 27 & 16 & 3 & 3 & 2 & - & - & - & 3 \\
\hline Mickens & 20 & 23 & mo. & & 352 & 226 & 70 & $3 I$ & I5 & 3 & 4 & $\mathbf{I}$ & 2 \\
\hline Tracy & $5 I$ & 23 & mo. & & 136 & 95 & 16 & 12 & 7 & 3 & $\bar{x}$ & I & 2 \\
\hline Moore & 21 & & yr. & & 475 & 306 & 92 & $3^{8}$ & 14 & II & Mis & el. & 14 \\
\hline Holden & I I & & " & & 483 & 285 & 107 & 34 & 29 & & & & 28 \\
\hline ' & " & & $2 "$ & & 399 & 230 & 90 & 37 & 17 & & & & 25 \\
\hline & " & & $2 "$ & B & 173 & 113 & 30 & 13 & 6 & & & & II \\
\hline Humphreys & I 2 & & $2 " 1$ & $\mathbf{G}$ & I I 2 I & 592 & 282 & $I_{13}$ & 56 & 35 & 28 & 6 & 9 \\
\hline Tracy & $5 I$ & & $2 "$ & & 263 & 168 & 52 & 22 & 7 & 7 & 4 & - & 3 \\
\hline & $" \%$ & & $2 "$ & $\mathbf{B}$ & I39 & 95 & 21 & 6 & 4 & I & - & - & 12 \\
\hline “ & “ & & $2 " 1$ & G & 285 & 187 & 46 & 30 & I I & 3 & 5 & 一 & 3 \\
\hline$"$ & “" & & $2 " 1$ & G & 36 & 16 & 7 & 4 & 3 & 3 & - & - & 3 \\
\hline Gale & 7 & & $2 "$ & $\mathbf{G}$ & 436 & 232 & 99 & 49 & 27 & 12 & 8 & I & 8 \\
\hline ، & $\therefore$ & & $2 "$ & $\mathbf{B}$ & 115 & 69 & 17 & 17 & 8 & - & 2 & I & I \\
\hline$"$. & "“ & & $2 "$ & $\mathbf{G}$ & 578 & 293 & 129 & 70 & $3^{8}$ & 27 & 13 & 6 & 2 \\
\hline Jegi & 13 & & 21 & $\mathbf{G}$ & I 227 & 727 & 238 & 139 & 54 & $2 I$ & 24 & 4 & 20 \\
\hline Gale & 7 & & 24 & & 400 & 247 & 82 & 36 & 23 & 4 & 4 & - & 4 \\
\hline . & $\therefore$ & & $2 " 1$ & B & 729 & 417 & 171 & 68 & 36 & IO & 9 & - & 17 \\
\hline " & 1 & & $2 "$ & & 741 & 395 & 159 & 97 & 27 & I I & 4 & 一 & 49 \\
\hline r. & " & & $2 "$ & $\mathbf{G}$ & 783 & 555 & 103 & 70 & 27 & I 2 & 4 & - & 12 \\
\hline Mickens & 20 & & $2 "$ & & 427 & 256 & 94 & 40 & 24 & 6 & 4 & I & 2 \\
\hline Pelsma & - & & 21 & & 379 & 212 & 88 & 37 & $2 I$ & 8 & 6 & I & 6 \\
\hline Mickens & 20 & & mo. & . G & 507 & 302 & IOI & 53 & 33 & 8 & 6 & 2 & 2 \\
\hline Tracy & $5^{I}$ & & mo. & G & $25^{\circ}$ & 196 & 28 & I5 & 5 & 3 & 3 & - & - \\
\hline Mickens & 20 & 26 & mo. & . G & 587 & 335 & I 21 & 60 & 47 & I I & 8 & 2 & 3 \\
\hline Tracy & $5^{I}$ & 27 & mo. & . G & I7I & II 3 & 27 & I6 & IO & 2 & $\mathbf{I}$ & $\mathbf{I}$ & I \\
\hline Mickens & 20 & 27 & mo. & . G & 675 & 382 & 139 & 66 & 62 & I I & 9 & 2 & 4 \\
\hline Tracy & $5 I$ & & mo. & . $\mathbf{B}$ & 677 & 422 & 136 & 69 & 23 & 9 & 12 & 2 & 4 \\
\hline 10 & & 28 & mo. & & $45 I$ & 287 & 89 & 29 & I9 & 13 & 6 & I & 7 \\
\hline 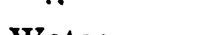 & & & mo. & & 327 & 132 & 87 & 42 & $3^{I}$ & I8 & 12 & 3 & 2 \\
\hline Watson & 8 & & mo. & & 480 & 257 & 124 & 50 & 21 & Io & IO & 6 & 2 \\
\hline Gale & 8 & 30 & mo. & . G & 769 & 444 & 149 & 103 & 30 & 19 & I I & 5 & 8 \\
\hline I & 8 & & mo. & . B & 1432 & 797 & 310 & I 54 & 75 & 33 & 25 & 10 & 28 \\
\hline "“ & 8 & 30 & mo. & & 1509 & 913 & 310 & 157 & $6 \mathrm{I}$ & I6 & I6 & - & 36 \\
\hline Salisbury & 23 & 32 & mo. & & 642 & $35^{\circ}$ & 150 & 60 & 32 & 24 & I 7 & 4 & 5 \\
\hline Gale & 7 & & yr. & & I I 76 & 675 & 238 & 143 & 53 & 33 & 17 & 7 & Io \\
\hline Whipple & 33 & & yr. & B & 1771 & 993 & 391 & 209 & 89 & 33 & 24 & 8 & 24 \\
\hline Pelsma & - & & yr. & G & $68 \mathrm{I}$ & 406 & 147 & 65 & $3 I$ & 14 & 9 & I & 8 \\
\hline Mateer & 19 & & yr. & B & I020 & $57^{8}$ & $2 \mathrm{II}$ & 125 & $5 \mathrm{I}$ & $2 \mathrm{I}$ & 4 & I2 & I8 \\
\hline Pelsma & & & yr. & & 1278 & 730 & 265 & 135 & 86 & 23 & 19 & 5 & 15 \\
\hline
\end{tabular}

V. COLOR VOCABULARY

Sanford (24) and Wolf (36) state that the average educated adult uses only about 25 color terms; and many fiveyear-old children are limited to four or five colors. The average two-year-old child uses only one color term. Preyer (45) states that his child knew no color terms at twenty months, and only red, yellow, black, violet and brown at 
PELSMA, JOHN R., A child's vocabulary and its development, Pedagogical Seminary, 17 (1910) p.328-369

A CHILD'S VOCABULARY

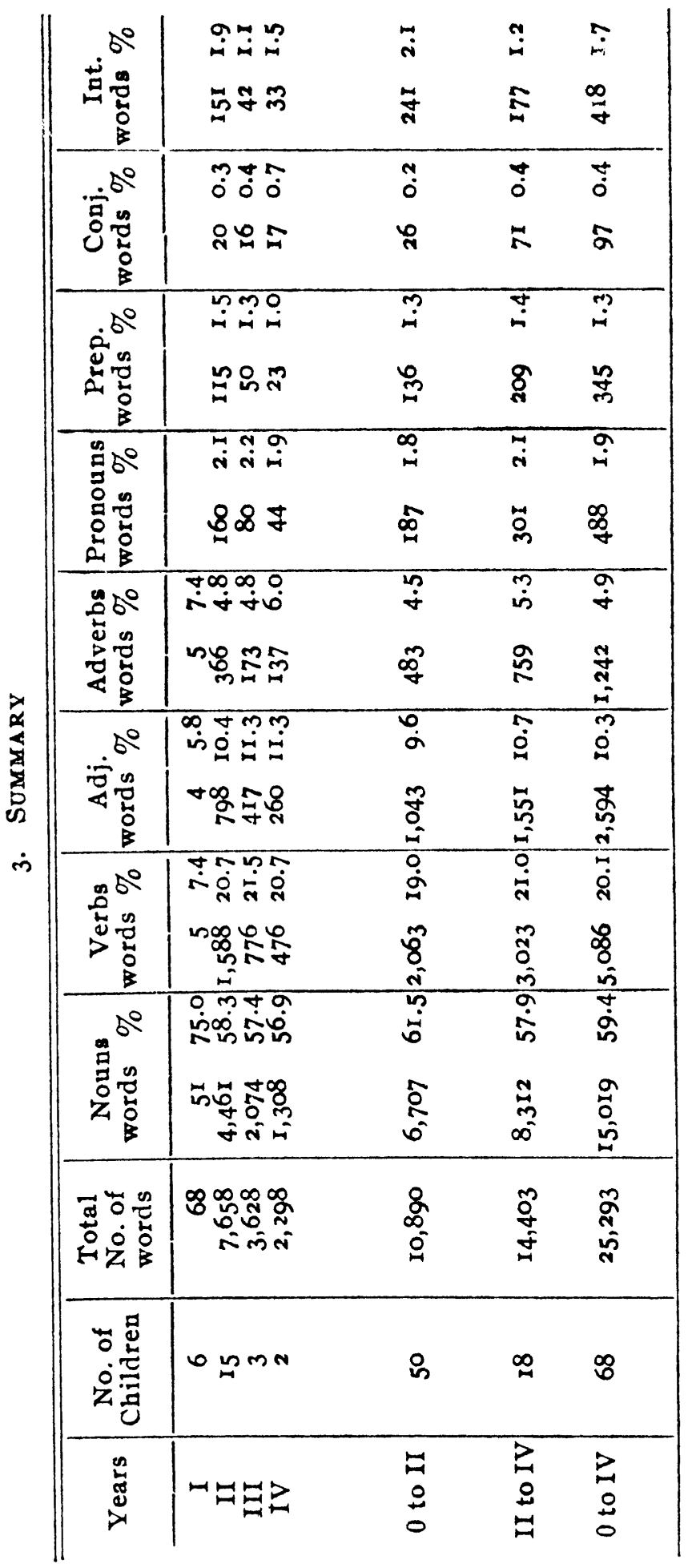


thirty-four months, and was not always sure of green and blue. Sanford reports one child as follows: At seventeen montbs, none; twenty-four months, blue, brown and pink; thirty months, black, white, red, green, blue, brown, pink; forty months, black, white, red, green, blue, and yellow.

"E" used no color terms at twelve months of age. Her first color term was blue in her eighteenth month. Her mother had put a blue dress on her. This greatly pleased her and she began saying "blue dress" in imitation of the expression used by her mother. By her twenty-fourth month she could distinguish the following colors and name them correctly: blue, green, red, white, and yellow-5 in all.

As her mother frequently worked with paints-painting felt and china, "E" soon became acquainted with color terms - pure colors, shades and tints. At her thirty-sixth birthday she used correctly the following terms: blue, black, brown, dark, green, gray, light, pink, red, white, yellow-I in all. This number steadily increased until at her forty-eighth birthday she was using: blue, black, brown, chrome-yellow, dark, dark-blue, dark-brown, green, gray, light-brown, orange, pink, purple, red, tan, white, yellow, yellow-ochre-18 in all.

This is greatly in excess of the number used by the average child, but is due to her unusual opportunity to associate almost daily with these colors. When her mother was painting she also desired to do so, and was very early given a brush and water colors.

\section{NUMBER VOCABULARY}

Whipple (32), Mateer ( 19 ), and a number of other writers attempt to justify themselves in inserting "fifty," "ninety," "one thousand," etc., in their list of words. The child may use them in his speech but certainly cannot comprehend their value sufficiently to justify their addition to the list of words in the vocabulary. No numbers have been included in " $E$ ' $s$ "' list above "six," for the same reason that words used in her nursery rhymes have been excluded-they have no content in the child's mind, not even magnitude. Number expresses relation. When 'E"' was asked which she most desired, I5 marbles or 20 marbles, she would hesitate a moment and then choose at random, showing that she did not comprehend any mathematical ratio.

At twenty-four months of age she could count to five, and by various tests made showed that she understood the numerical relations of "one," "two," and "three." It was with great difficulty that she was taught to count beyond five. Doubtless the "plateau stage" was here operative; but by her 
third birthday she could count objects to fifteen; and understood the integral relations of four. She would name four objects when presented to her without counting them. If asked how many two and two made, she would answer "four." She knew all these integral combinations without their concrete application; in fact, she preferred not to use apples or blocks, though her results were equally accurate with concrete application.

When forty-eight months old, she could count correctly twenty objects, and very seldom made an error. However her concept of number relations ended with "six." She knew that six was more than five and knew that it was one more. She could give all the integral combinations which made six. If told that there were six apples on the table and that four were removed, she knew that two remained. Concrete application seemed to be no aid to her. She knew the correct result as readily when asked to tell how much three and three would make, as if asked how many apples three apples and three apples would be. And as far as it was possible to determine she did not imagine apples or any other objects. It is possible, however, that she had repeated the answer over so often that the result was associated with the problem,-a mere memory process rather than an abstract logical concept of their quantitative relation; just as the child who learns that $6 \times 7=42$, without having formed any mental image whatever - merely a matter of associative memory.

This may, however, throw an interrogation into the theory usually advanced by educators that all number work in the first few grades of school should be concrete.

In comparison: A. Stevenson (27) reports a case in which the child could not use number terms beyond two until nearly in his fourth year. Any number beyond two was nine. Jegi's child ( 13 ) at two years of age counted to twelve, but could use only one and two correctly. Whipple's three-year-old boy counted correctly to thirteen, and could apply correctly numbers frow one to four (33).

\section{B. Development}

I. ORIGIN OF SPEECH

Settlement of this controvertible problem will not be attempted; neither shall we summarize present theories, nor advance a new. Philologists have long recognized the similarity between the development of the speech of the child and the language of the race. That children can invent a language of their own is now generally conceded by psychologists. Color is given to this view by examples cited by Dr. Hale (Romanes (47), Chamberlain (33), Stumpf, et al.). Children, 
however, have this advantage over the race-an example has been set for them, and through the process of evolution have come into the world with a richer inheritance of speech organs and speech centres than primitive man. Still this process may be the same in kind if not in time. Parents imitate children and thus put meaning into their "lol" words. This aids materially in shortening the time.

The "Divine origin theory" made its exit at the advent of the theory of evolution. The "Onomatopoetic" (Darwin), Ejaculation (Whitney), and the "ding-dong" (Max Müller) theories have each much in their favor. That some words are acquired in each of these ways may be corroborated from the vocabulary of every normal child. But these theories fail to explain how the race acquired the power to use its imitations and associations for speech purposes. Much might be said in favor of Ludwig Noire's theory that a man thinks because he speaks, rather than that he speaks because he thinks. But why not a parallel development? Why must one be necessarily the sequence of the other?

A child's early sounds and babbling are not directed with a conscious purpose to convey thought. (Wundt (54)). But when the child cries "ma" and "da," the proud mama and daddy feel flattered and imagine the baby is learning to speak, come to it and joyfully repeat the sound, minister to its wants, and thus associate themselves with the sound uttered. The child consequently by its usual habit of repeating a pleasing sound, expands "ma" to "mama," and "da". to "dada." These early sounds are not uncommon in the animal kingdom -example, the lamb. "Ma, da, la, ta" become root words in many languages (Chamberlain (35)). Again, the child is a product of his social environment. His emotional nature is not unlike that of the adult. His social environment aids and stimulates him in expressing his social needs. The development of language in the human race is synchronous with the advent of the gregarious instinct. Mutual sympathy and sinilar emotions led to the expression of this common feeling. Later, intellectualization on the part of the child and the race permitted ideational processes resulting in abstract language or language proper.

Observation of ' $E$ "' illustrates the foregoing processes and stages. This will be elaborated under II, 1 , and 2 , sufficiently; a similar statement at this point would be redundant.

\section{Growth. I. Stages}

a. Reflex. Psychologists in general agree that the different stages of acquiring a language may be to a certain extent chronologically classified, though all stages overlap irrespec- 
tive of the basis of classification. There is, too, a general agreement that the first cry of an infant has no language significance. Preyer (46), Perez (44), Moore (2I), Trettien (3I), Sully $(50)$ and others agree that it is a cry of pain, rather than "a cry of indignation and of wrath" (Kant), or "a lament over the sin and misery of this world." How may we be sure that it is even a cry of pain? Preyer (46), Shinn (49), and Genzmer state that the newborn child is insensible to the prick of a pin, application of ice-cold bodies to the skin, and even goes to sleep under a minor operation. "New-born children without any brain at all cry out" (Preyer (45)). Some instead of crying, sneeze (Darwin). Preyer (45) contends that though a child is inseusible to local peripheral stimulation, pain may come from a feeling of general discomforture, $i$. e., a change of temperature; and that the expression on the face of a child at birth showed that it was in distress. We have no right to interpret expressions of a child as resulting from sensations from the standpoint of adult psychology. Comparative psychologists certainly do not grant us this privilege. Since children without brains cry out, we may do well to look for some other cause. Sigismund says that the sound is made by the air rushing into the lungs. This looks logical. Peripheral stimulation affecting a partly developed nervous organism produces a teusion of the vocal chords, and the inrushing air produces the cry. In the case of " $E$ "' it was a distinct wail which continued for a few seconds, and seemed rather mechanical, like a toy which gives a sound when a spring is touched.

Neither have the profuse spontaneous gestures any language siguificance at this period. The gestures are not physical expressions indicative of psychical activity. The child has no thought or emotions to express (Preyer (46), Tracy (5I)). His senses have not yet awakened. $\mathrm{He}$ is blind, deaf and insensible to other local stimulation. This stage may be adequately termed the Reflex stage. (Lukens (I7), Kirkpatrick (I5)). For the first few weeks the vocal expressions and physical gestures result from external stimulation, and not from mental initiative. At this stage the gestures are profuse and undirected. As every impression must have an appropriate expression (Judd (38)), the sensory stimulus which the child receives is diffused when it reaches the central nervous system and sets into activity the muscular phenomena observed in every child. These gestures are meaningless as far as an expression of inward emotions or concepts. A pleasurable or painful sensation may cause violent dangling of feet and arms, but it is neither directive nor differential.

b. Cry and Gesture Stage. After the first few weeks the cry is differentiated and the gesture directive, and characteristi- 
cally expressive. This is the period which has been poetically and likewise scientifically expressed as, "a crying in the night, crying for the light, with no language but a cry." Miss Shinn (49) becomes quite poetical when observing the cry of her niece, and proceeds: "Oh, cry of the baby in the night, nightingale for father and mother." Perhaps. I suppose I am rather prosaic; but to me it resembled more nearly a firealarm-a hurrying to and fro through cold halls, seeking for mislaid paregoric bottles, etc., etc.

M. Egger states that the voices of children in earliest infancy are not characterized by an individual timber. This is stoutly denied by Dr. Laurent and Perez (44). Tracy (5I) states that the cry at first is a reflex squall without expressive modulation or distinctive timber. The same cry serves to express all sorts of feeling. "E's" mother could easily distinguish her cry from many other infants after the first week. At the end of the second week "E's" cry had two inflections -one for comfort and one for discomfort. At the end of the fourth month these cries were sub-modified, for multiple wants desired expression. Preyer (46) states that the word "atta" had at one time eleven different meanings which were expressed with modification and appropriate gesture. The cry of hunger is one of the first to manifest itself. M. Taine (29) says that at this period "all shades of emotion, wonder, joy, willfulness and sadness" are expressed by difference in tone. Sully (50) states, "This cry gradually differentiates itself into a rich variety of expressions of hunger, cold, pain, joy, etc." "The cry of pain," says Leonard, "is generally longer continued than the cry of fear." Hunger is often expressed as a prolonged wail and the cry of fear as being more abrupt and explosive. Lukens (I7) states, "the long drawn-out cry of hunger, the subdued and monotonous sleepy cry, the short, high-pitched yell in response to some physical want, and the loud cry of anger, seem pretty well characterized." Mrs. Moore (2I) records that "we can distinguish the sounds of hunger, sleepiness, anger, and joy at four months of age."

"E's" cry of pain was a sharp, long, piercing cry. Her hun. ger cry at this period was a repeated vocal utterance "äuă" and rot as shrill as the cry of pain. Contentment was expressed by a droning coo. Anger, or general discontent, or "spunk" was noticed at the end of the fifth month. This cry was harsh, somewhat guttural, and otherwise characterized by a violent gesticulation of bands and feet, with extreme rigidity of the rest of the body. The motion of the hands and legs appeared to express repulsion. This manifestation has changed but little during ber four years of life. What Sully calls "fear" was expressed by an attempt to shrink away, the corners of 
the mouth were pulled down and short broken cries followed. All these physical manifestations are indicative of emotional conditions. Negation was expressed by ' $\mathrm{E}$ '" as early as the third week, by a turning away of the head when refusing more food. Desire for objects was indicated by an extension of the hands in her 18 th week. These expressions should be classed as the rudiments of gesture language, which precedes vocal expression as a means of communication (Judd (38) ).

c. Babbling. During the thirteenth week " $\mathrm{E}$ "' began to modify her beretofore purely vocal and labial sounds by lingual sounds. She chanced to discover that agreeable sounds could be made by wiggling her tongue. She seemed to take great delight in lying on her back and singing "nannannan" and alalalala, etc. Both the melody and the muscular stimulation produced a pleasing sensation. This meaningless babble cannot be called speech. That it is entirely free from imitation is not beyond all doubt; but the evidence points to the fact that all normal children will produce similar sounds (Sully (50) ). It doubtless presents a chance discovery; it stimulates the child agreeably and the sound is repeated again and again, frequently with considerable modification in pitch and intensity. Mrs. Moore (2I) observed that by the close of the fourth month her child had produced nearly all the elementary sounds. The child begins with the vowel sounds; then appear the consonants in combination with vowels (Meumann (4I), Perez (44), Shinn (49), Preyer (46) ). Since "speech is the use of a sound intentionally as the sign of an idea" (Sully (50)), this babbling is not speech; for we cannot believe that an idea accompanies all this babbling. Yet true language sounds significant if ideas develop from this spontaneous expressive articulation. Buckman (3) puts much confidence in what he calls the ma-data-la series. Muscular co-ordination and control of tongue and lips is here so far perfected that true language may be said to appear in the next stage, viz.:

d. Imitation. This stage, according to Tracy (5I), embraces the second six months of the child's life. The simple imitation is observed quite early, conscious imitation appears first between the seventh and ninth month (Preyer (45)). Gestures, too, are now consciously imitated; are no longer mechanical, but result from an effort to experience how it "feels" to perfrom an act, or see if he, too, can do it. "The child desires the experience which comes from performing the thing he sees others doing. He wishes to increase his experience," (King (39) ). The expression of satisfaction of a child at the success of his efforts leaves little doubt as to his 
purpose. His first successful efforts at real articulation come during this stage. The child learns the language of his parents and the peculiar characteristic accents are to some extent acquired. That the child attaches a definite meaning to his articulations results greatly from his parents imitating him and associating a definite percept with the sound. (Wundt (55) ). "E"' would hear a chick and she would say "peep" or more correctly "e" and then "coo" at her success with a jumping motion of the body indicating satisfaction. She would listen to the clock and say "ic-ic," or attempt to repeat many of the elementary sounds in imitaion of her parents. " $E$ "' imitated many words quite perfectly ; would say mamma, papa, boy, dog, with a distinctness highly gratifying to her parents, who were quite elated over the linguistic progress of their first offspring, when the next stage appeared and, in the language of Shakespeare, "Ob, what a fall was there."

e. Plateau Stage. With apologies to Bryan and Harter, (Psych. Rev., Vol. VI, p. 346), we believe the above term expresses adequately this period. It represents a time of accumulation and co-ordination, of assimilation and adjustment. There is interpretation with little verbal expression. Progress is made, but very slowly; This stage usually appears between the ninth and fifteenth month of the child's life. Frequently the energy seems to be directed into other channels, viz.: teething and learning to walk. If the child has already learned to walk, or acquired this ability in a day (Jegi (15)), this plateau stage may not be very pronounced. In fact, it is frequently so shortened and modified that it is not readily observable (Jegi (13) ). In other instances it is exceedingly pronounced. Lukens ( 17 ) cites a very interesting case. Such stages are not infrequent in other fields of psychical and physical adjustments (Judd).

After "E" used the words boy, dog, papa, mamma, with various application, but with rather distinct enunciation, about the tenth month, there was little progress in articulation until about her sixteenth month. "Boy" meant almost anything from a cow to a fire-shovel. If she saw a cat she would say "boy." If she saw a house it was "boy." The inflection, however, varied. But it could not well be observed whether the same inflection accompanied similar objects. Later the word "dog" or rather "da" was most favored, and at least every moving thing was "da." By the end of the twelfth month "boy" had become restricted at least to animate objects; "dog" was seldom applied to any other animals but a yellow hound, a billy goat and a pet brindle cow. The remaining eight words she used have been 
explained. Gestures were freely indulged in, and she would turn her head toward many objects mentioned to her, and would pick up objects from the floor if told to do so; but she could not be coaxed into attempting to pronounce them. A few words as "doll" and "bye-bye," which she had been accustomed to pronounce quite distinctly, she no longer attempted in her twelfth month. These words were not regained until her fifteenth month. She learned to walk in her twelfth month. This may have aided in directing her energy into another channel, but it does not explain the whole problem. She was on the "plateau" of development. Trettien (3I) states that a child, "F," at eleven months had 80 words, which she had securely associated with ideas, while her spoken vocabulary was very limited.

f. Expression. When psychical and motor adjustment of speech centres and organs is nearing completion, the child makes rapid progress in acquiring its native tongue. Trettien's ( 3 I) child " $F$ " at eighteen months made very rapid progress, imitating nearly every word proposed to her. Mrs. Hall (ro) states that her child in its sixteenth month acquired 93 new words, an increase of 45 over the previous month. Mickens (20) states that at 22 months of age his child acquired 70 new words, an increase of 46 over the previous month. Trettien (31) writes, "Felix was very backward in learning to speak. Suddenly, one evening, it was as if the Holy Ghost had descended upon him and had poured into him the gift of speech; for he repeated everything that was spoken to him very correctly." These few examples clearly show that when the "plateau" stage has passed rapid progress is made. Like learning to walk, this ability may be manifested in a day (Kirkpatrick (40), Jegi (13), Lukens (17) ). "E"' made little progress until her eighteenth month; then she seemed to take special delight in imitating nearly all words she heard. She would pronounce words often which surprised us greatly, not remembering when she might have heard them. This was her last stage. She was now in line to become a full-fledged linguist.

\section{METHOD}

More important than the stages, is the knowledge how the child gets its vocabulary. The method is difficult of analysis; (I) in the beginning it must be wholly by external observation, (2) later this may be supplemented by a more subjective process of questions and answers, and experimentation. But this requires the following precautions suggested by Meumann (4I): (I) The child's vocabulary must be interpreted in the psychology of the child. (2) The interpretation of the words 
must be studied from the standpoint of genetic psychology. (3) No abstractions occur in his vocabulary (this point is not conceded by all). (4) Observations should be extended to the fifth or sixth year, as these latter years aid in interpreting the first few years. The first precaution cannot well be over em. phasized. Adults are apt to "read into" the child's activity many qualities from their own viewpoint.

a. Reflex Expression. Confining ourselves to vocal expression, the first cry of the child is reflex. It is not emotional. "It seems altogether impossible that the expression of the new-born infant, however violent, can have any emotional significance." (King (39) ). His cries come from external stimuli or from internal organic adjustment. The cry is not of discomfort; it is instinctive; it is but the adjustment of "impression and expression" - a matter of co-ordination of stimuli and motion, not emotion.

b. Spontaneous Emotional Expression. The first method is the precursor of the second, yea, more, a determinant. Gradually the child becomes conscious of the spontaneous, impulsive, instinctive activity. The machinery is being adjusted; "A living, conscious automation," as Miss Skinn (48) interprets it. Sensations are inhibited, cognized, and directed into dcfinitely established channels. The efficiency of this adjustment gives a general emotion of pleasure; when ill-directed or momentarily inhibited there is a conflict and the emotion is pain. The expression of fear often observed may be only the maladjustment, or the necessity for new adjustment of stimuli and response. Fear then would not be the result of heredity or education as Preyer (46) notes, but the kinæsthetic memory of the prior emotion resulting from a former mal-adjustment, or rather a tendency to check instinctive responses to social stimuli. King (39) believes that a child is born with a small number of motor co-ordinations of an instinctive type, and any stimulus may be diffused and find expression in a variety of avenues-joy, fear, etc., and the direction the stimuli take is dependent largely on what channel has been previously used. But the conflict does not always remain painful. The child early differentiates the emotions. The conflict may then manifest itself in wonder, surprise, joy, etc. Out of this emotional attitude of response arises the first exclamation of articulate language. His vocal expression of these emotions may be limited to a few syllables, for vocal expression is dependent on physical adjustments which have not yet sufficiently matured to respond to a great variety of inter-reactions or combinations of sounds. When the child points to an object and says "da" (the same in many languages) it is, probably, in its inception merely an interjectional expression of the "faint shock of won- 
der produced by the appearance in the visual field of a new object" (Sully (50)). As fast as the child can adjust his vocal organs to his emotions we have various expressions. These are at first more related to singing than talking (Sully (28)). This may have been true of the race (Darwin).

c. Volitional Expressions. When a child becomes conscious of its activity and able to direct expressions at will, he can make a choice. Perez (44) states that it is manifest at first simply in the substitution of one tendency for another resulting from conscious attention to reflexive activity. James says that this cannot be until the child is capable of memory images, Wundt (Menschen-und Thier-seele), on the contrary, maintains that attention is present in the most mechanical movements. The child remembers the experience of former activity and desires again to experience this sensation and he says "yem" when he desires to get down for a buggy (Grossmann). " $E$ "' very early acquired the word "down" and "uppaday" when wishing to get down from or up on my lap.

She said "i") when she desired water and "ک" when she wished to nurse. At the age of 27 weeks Sully's (50) little boy said " $o$ " for the expression of pleasure and " $a$ " for surprise. Darwin's boy said "mum" when it wished something to eat. These first articulations are all expressions of emotions (Meumann (40)) and are consciously directed. They may be designated as "wish-words" or "feel-words."

d. Imitation. This method was partly outlined when discussed under (II-I-d). Preyer (46) writes: "Imitation is without doubt the first and most important factor in the learning of language by the individual." Sully (50), says "Learning the mother tongue is essentially a kind of imitation." When the child has developed his muscles by promiscuous kicking and dangling, he gets up and walks. When he has been babbling for some time and developed and co-ordinated his speech apparatus, he begins to "talk" - begins to imitate sounds heard and associates with this sound definite mental concepts. He has developed the power of active attention sufficiently to enable him to reproduce sounds spoken in his presence. "He imitates the sounds made by animals and inanimate objects" (Preyer (44)): more than this he will at this age (twelve months) attempt imitation of physical expression of emotions. When sounds and expressions are imitated, the child associates a meaning not only with the auditory sensation, but the kinæsthetic as well. " $E$ '" exhibited no abnormal peculiarities in this respect. She observed carefully gesture and articulation of her parents, would repeat what she heard, and sing when others sang.

e. Associative-Reproduction. When the child begins to in- 
tellectualize (Meumann (40)) he begins to analyze and differentiate. "Papa" no longer stands for the expression of an emotion but for an idea. Certain attributes are recognized as belonging to a definite object. "Papa," at first, was an expression of pleasurable emotion which was uttered when father came home; or unfortunately it might be the expression of a painful emotion. Now, "papa" represents an intellectual process-an ideation. At first "papa" has attributes in common with other objects-all men are "papa." Perhaps the child may be capable of more accurate discrimination than usually credited with when he calls men papa, but since his vocabulary is limited, he may find it convenient to so express the classification just mentioned. The word "papa" most nearly expresses his concept. Meumann (4I) thinks that the child does not form general concepts until about four years of age, and it is doubtful whether distinct classification appears much earlier, but the associative process is developed much earlier (first six months). When he acquires the word "man," a concept distinct from "papa," he at least begins analysis. "Man" now becomes a general concept and "papa" is again applied to a specific object. But this "papa" is an altogether different "papa" from the former two. The first was a mere percept. The second a concept now expressed by "man;" the last a concept, though doubtless very limited in its application.

When the child imitates he reproduces but there is no associative-reproduction. At first they are "pennies," then some become "white pennies." " $E$ " saw a duck and said "quack." The duck is in a latticed box, and the box is also "quack." She found the picture of a goose in a story book, and the book henceforth became a "quack" book. When in her third year she had observed her father "run" rats in a "maze" at the psychological laboratory, his co-worker, a fellow student, was always present on such occasions. When " $E$ " would meet this man on the street she would always exclaim: "There comes the rat-man." She had been told that a certain big yellow dog had been found at a fire. She often begged her father to get her a "fire-dog" Lather she would call "shave;" would say "hatchet that for me," meaning, "chop that with a hatchet." The teacher who collected the pennies at Sunday school became her "penny-teacher," and the candy secured from an automatic street-vending machine, was called "punch-candy."

Associations are easily formed. The child is a poet. He can see likeness and similarity where the adult observes only differences and contrasts. Associations are primarily spatial, although they are at times syuchronous, rarely attributive in the first few years. Inferences are rarely logically drawn before the age of four. Induction is prior in development to deduc- 
tion; yet who is the psychologist so bold as to claim to have fathomed the process of a child's mind? When " $E$ "' was four, one day while passing along the street she noticed a tree which had been cut. She asked if George Washington lived there. She had been told the story of the "hatchet" in her kindergarten, but had never mentioned it in her home. Was this deduction or mere association? Frequently in attempting to read the efforts at psychological analysis of the mental activity of an infant child we are reminded of the answer given to the question of old Polonius: "What read you, my lord?" "Words, words, words!"

$f$. Invention. If children were forced to live alone would they develop a language of their own? "Yes" say Judd (38), Tanner (52), Sully (50), Tracy (51), Romanes (47), Taine (29), Preyer (46). "No" say Wundt and Idelberger (55). "Perhaps" say Meumann (4I), Compayre (37), et al. Nearly all admit that the child is normally not permitted to exercise his inventive genius to a great degree, also that too much credence cannot be given to the stories of Dr. Hale and others. Experimentally we are much in the dark, as yet. That normal children never invent words, both sound and meaning, is difficult to disprove, but without imitation there would be little resemblance to a Yankee vocabulary. Preyer (46) states that "after sound and sense is once established through imitation," the child then freely invents new combinations, "although to a more limited extent than is commonly assumed." Much of the disagreement here is the result of the definition "inventing articulate speech." Methods of invention are unique and varied. "E's" invented words may be roughly classified thus:

(I) Borrowed from adults but with wrong form. A pure creative element is not found in child speech (Meumann (4I)). The influence of the adult begins at birth. Infants who early associate much with their parents learn to talk sooner than those who do not have this privilege. Since examples of invention might be multiplied in profusion, illustrations will be drawn only from our own observations. In her fourth year she used the word "thernly." An object was not a "thernly" bit nice. This doubtless came from certainly. She often told us not to "mickle" her things. This may have originated from mix up or muss up.

(2) Proper words with wrong meaning. This introduces the disputed subject of classification of parts of speech from a child's standpoint. Miss Shiun (49) states that nouns and verbs are always being interchanged. " $E$ " used "hatchet" for "chop;" "smoke" for "pipe;" "corns" she called "popcorns." She at first said, "sugar-bunch," then "sugar-hump," next "sugar-lump." She frequently said, "Don't tell me any more questions." 
(3) Onomatopoetic sounds. Every normal child will naturally call a dog a "bow-wow," a clock a "tic-tic," and a cow a "moo," etc. " $E$ "' imitated three sounds very accurately, and other similar sounds without much practice. Many of the sounds imitated never became embodied in her vocabulary. Much of the imitation is for the pleasurable emotions furnished.

(4) Onomatopoetic sounds otherwise applied. Through association these sounds are applied to other objects. "E" called a latticed box a "quack" because the ducks within had been so designated.

(5) Ejaculations. Natural grunts, and other vocal utterances are frequently attached to objects, or actions. Example, "ouch," "'boo," “oh," "ōo," etc. Emotions are often thus expressed.

(6) Construction from "lol" words. Children not infrequently construct some original word from their early babbling sounds. Many of their first words are such. Some contend that the child attempts to imitate here, but is unable to reproduce the sounds he hears. Should this always be true, it fails to explain why he continues using it after being able to articulate more distinctly. " $E$ " used "I" for water, "uppeday" for up. "Da" meant "daddy," "there," and many other ideas. "Rolabola," is still being used.

(7) Combinations. All sorts of combinations are made by the average child; thus coining new words. He sees similarity between quite incomparable objects. "E"' seeing a small coffee-grinder, called it a "coffee-churn." Candy obtained from automatic slot-machines was called "punch-candy." The teacher who gathered up the pennies at Sunday school becawe her "penny-teacher." A boiled egg was a "cup-egg;" a fried one a "plate-egg." A sack with holes was a "hole-sack," a yellow dog, a "fire-dog;" a conductor, a "ticket-puncher."

(8) Formation of regular variants. Irregular inflections of verbs and adjectives are difficult for the child. " $\mathrm{E}$ " invariably' said "Bleeded," "hurted," "rund," " seed," "gooses," "tooths," and the like. These may not be pure inventions, but they are formations which the child has not heard, but creates from a knowledge of the laws of regular inflections.

\section{Systematic Classification of Progress Madi in Different Parts OF SPEech}

The following table shows the progress made in each suidivision of speech. It is self-explanatory. It may be interesting to observe that not all the words which appear one year are found in the next.

All of the first year words appear in the second. 
5 nouns, 4 verbs, 1 adjective, I adverb, and I interjection found in the second year are not found in the third.

24 nouns, 2 verbs, I adjective, I adverb, I preposition, and 4 interjections found in the third year are not found in the fourth.

All of the second year words appear in the fourth.

"Looking-glass" in the third becomes "mirror" in the fourth. "Night-drawers" in the third becomes "night-gown" in the fourth. "Sew" is found in the third, but "sow" in the fourth. "Teeth" is found in the third, "teeth" and "tooth" are found in the fourth. These illustrate some of the changes continually going on.

Gale (7) records the following summary: 35 nouns, I 6 verbs, 4 adjectives, I adverb, which were found in 'Ruth's' vocabulary at two years of age and not found at three.

Tabolation

\begin{tabular}{|c|c|c|c|c|c|c|c|c|c|c|c|c|c|c|c|c|}
\hline \multirow[b]{2}{*}{ Age } & \multicolumn{4}{|c|}{ Nouns } & \multicolumn{4}{|c|}{ Verbs } & \multicolumn{4}{|c|}{ Adjectives } & \multicolumn{4}{|c|}{ Adverbs } \\
\hline & $\mathbf{I}$ & II & III & IV & I & II & III & IV & I & II & III & IV & I & II & III & IV \\
\hline Total & 7 & 212 & 406 & 730 & 1 & 88 & 147 & 265 & & 37 & 65 & 235 & 2 & $2 I$ & $3 I$ & 86 \\
\hline $\begin{array}{l}A \\
B \\
C \\
D \\
E \\
F \\
G \\
H \\
I \\
J \\
\mathbf{K} \\
\mathbf{L} \\
\mathbf{M} \\
\mathbf{N} \\
\mathbf{O} \\
\mathbf{P} \\
\mathbf{Q} \\
\mathbf{R} \\
\mathbf{S} \\
\mathbf{T} \\
\mathbf{U} \\
\mathbf{V} \\
\mathbf{W} \\
\mathbf{Y}\end{array}$ & $\begin{array}{l}I \\
I \\
2\end{array}$ & $\begin{array}{r}7 \\
28 \\
26 \\
9 \\
4 \\
12 \\
8 \\
9 \\
2 \\
2 \\
4 \\
6 \\
12 \\
7 \\
2 \\
17 \\
1 \\
7 \\
24 \\
17 \\
2 \\
1 \\
5\end{array}$ & $\begin{array}{r}9 \\
48 \\
50 \\
14 \\
8 \\
17 \\
12 \\
18 \\
7 \\
3 \\
8 \\
15 \\
18 \\
10 \\
6 \\
43 \\
1 \\
14 \\
56 \\
29 \\
2 \\
3 \\
15\end{array}$ & $\begin{array}{r}10 \\
68 \\
83 \\
32 \\
18 \\
28 \\
18 \\
36 \\
9 \\
6 \\
11 \\
28 \\
38 \\
19 \\
8 \\
71 \\
3 \\
29 \\
110 \\
55 \\
3 \\
4 \\
42 \\
1\end{array}$ & $\mathbf{I}$ & $\begin{array}{r}2 \\
8 \\
10 \\
6 \\
1 \\
5 \\
3 \\
2 \\
3 \\
1 \\
2 \\
4 \\
2 \\
1 \\
6 \\
1 \\
4 \\
9 \\
9 \\
2\end{array}$ & $\begin{array}{r}3 \\
17 \\
14 \\
12 \\
2 \\
7 \\
6 \\
8 \\
3 \\
1 \\
3 \\
6 \\
3 \\
1 \\
1 \\
13 \\
1 \\
6 \\
17 \\
10 \\
2 \\
11\end{array}$ & $\begin{array}{r}3 \\
19 \\
19 \\
10 \\
33 \\
12 \\
7 \\
15 \\
4 \\
2 \\
5 \\
13 \\
15 \\
4 \\
33 \\
20 \\
1 \\
11 \\
51 \\
16 \\
6 \\
26\end{array}$ & & $\begin{array}{l}2 \\
2 \\
3 \\
1 \\
1 \\
2 \\
6\end{array}$ & $\begin{array}{r}3 \\
10 \\
3 \\
3 \\
1 \\
7 \\
3 \\
4\end{array}$ & $\begin{array}{r}9 \\
5 \\
7 \\
4 \\
4 \\
\\
5 \\
12 \\
15 \\
1 \\
\\
8 \\
2\end{array}$ & I) & $\begin{array}{l}4 \\
4 \\
I \\
I\end{array}$ & $\begin{array}{l}4 \\
5 \\
1 \\
1 \\
2 \\
1 \\
I \\
I\end{array}$ & $\begin{array}{r}\text { I I } \\
7 \\
2 \\
2 \\
4 \\
4 \\
4 \\
3 \\
1 \\
1 \\
1 \\
3 \\
3 \\
4 \\
7 \\
2 \\
2 \\
2 \\
5 \\
8 \\
2 \\
1 \\
4 \\
3\end{array}$ \\
\hline
\end{tabular}

III I. PronunCIATION

Indistinct articulation may result from the following causes: ( I) Undeveloped organs of speech. A child sometimes does 6 
A CHILD'S VOCABULARY

TABULATroN-Continued

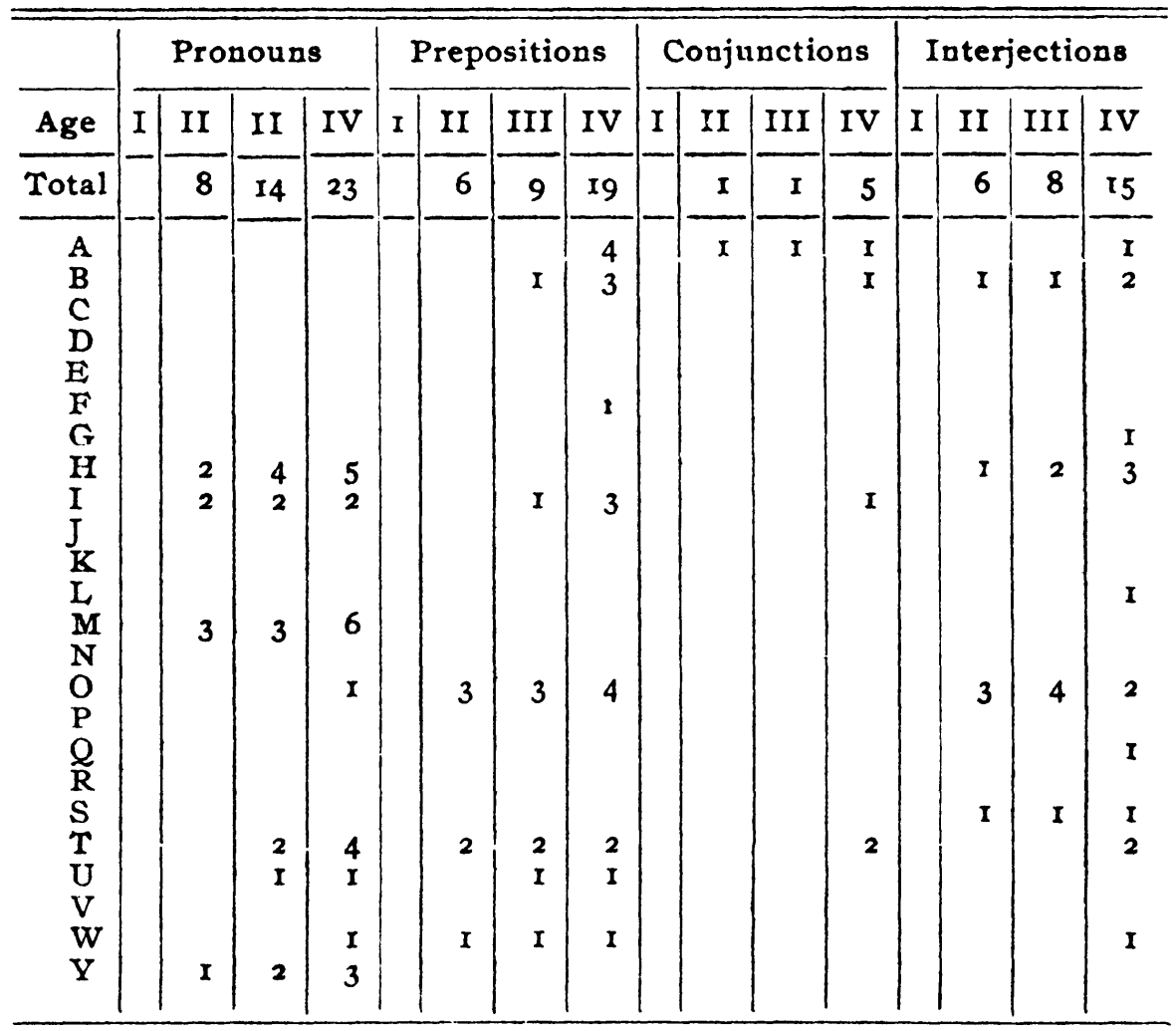

not articulate correctly because of lack of proper co-ordination of his vocal apparatus (Tracy (5I), Preyer (46)). His speech centre in the cerebrum is yet undeveloped. He is unable to manipulate the tongue, vocal chords, etc., to produce the sounds he may hear. "The power to understand words is usually more quickly gained than the power to control the vocal apparatus"' (Kirkpatrick (15)).

(2) Imperfect perception. Lack of discriminary power often prevents distinct enunciation. The auditory perception is undeveloped, and so the child says " $\bar{e}$ " for eat, and "tention" for attention.

(3) Lack of memory. Frequently the child hears a word and sometime later when he attempts to pronounce it he omits part, modifies, or substitutes. This is true, not of the child alone, as the following caricature from an English paper illustrates. A lady walked into a judge's office and inquired if he was the reprobate judge, and said that her husband had died detested, leaving her two small infidels, over whom she desired to be pointed executioner. ' $E$ " one day said she wanted 
a "Pantograph." This was the name of a local newspaper. She wanted a "pantograph with a horn." She had been visiting and had seen a phonograph.

(4) Imperfect example. Adults intentionally omit or slur certain sounds of words. The child reproduces only the accented portions, or substitutes more familiar sounds.

(5) Difficult combinations. Although certain elementary sounds or words may be distinctly uttered by the child, certain words or sentences containing these sounds are difficult to speak because of their combination. This tempts him to substitute another sound, as "wun" for run, "ting" for sing.

Noble (22) has given us a law of pronunciation, viz.: Correct pronunciation depends on close perception and knowledge of the motions necessary to produce sounds. Hence those sounds most easily articulated are labials and dentals; those most difficult, the gutturals and medials. Tracy (5I) gives the following order, beginning with the most difficult.

$r$ e th $v$ sh y $g$ ch $s$ ef $t \mathrm{nqdk}^{\mathrm{k}}$ ow a h m p b

Merely considering the initial sounds, though this is not an accurate criterion, the least difficult sounds for " $E$ "' were :

Second year K, B, S, P, T.

Third year $S, K, B, P, T$.

Fourth year $S, K, B, P, T$.

\section{Development of Correct Pronunctation and} Distinct EnUNCIATION

Since imitation is the important source of a child's vocabulary, it surely behoves parents and others to permit children to hear only correct pronunciation and distinct articulation. That some parents still believe that a child can better understand its own "baby talk" than the "Queen's English," is too true. Accuracy in pronunciation can result only from imitating correct articulation. The practice of using "baby talk" when speaking to children is decreasing. Preyer (46) thus gives his opinion: "Children should never at the expense of their own natural development become entertaining playthings to pass away the time for adults." Children who associate much with adults make more rapid progress in correct pronunciation than those who associate much with other children. " $E$ 's" articulation has always been quite distinct, barring natural hinderances caused by undeveloped speech centres and muscles of articulation. At the age of four there is no "baby talk," and her enunciation is as distinct as that of the average adult, except that the "'s" sound is still inclined to "th." Sit is "thit." But when shown where to place her tongue, the "th" disappears. The difficulty is found only in the initial "s." 


\section{SenthNCE Formation}

The first expressions of the child should be called sentencewords rather than "parts of speech." It is the whole of speech. When "E" cried "ma," it was a complete thought expressed. Meumann (4I) calls it a wish-word. It is rather a "wishsentence." As Waitz says, "The unit of language is not the word, but the sentence." And Romanes (47), says "every word was originally itself a proposition." Dewey (6) states that his child at twelve months of age had 17 words, but these were classified grammatically rather than psychologically. " $E$ '" at twelve months had ro words (see table) with similar classification. Though unpsychological there is no better method. Trettien (3I) states that such words (?) as "ma" (mother) should be called "interjectional - nominal - adjectival - verbal expressions. Dewey (6) says "door" is a nominal-adjectivalverbal-interjectional complex. This may be psychological and again it may be nonsense. Lukens (17) comes nearer being correct when he states that a child does not really use "parts of speech" in a grammatical sense until all parts of speech are used. "Parts of speech" are primarily grammatical. To quote Herbert Spencer, "Language was made before grammar." As with the race so with the child, "the sentence precedes the word" (Sully (50)). There is no universal psychological arrangement of the words in a sentence (Wundt (54)). Children like savages blurt out a complete sentence at once (Chamberlain (35)). Early Roman sentences were not divided into words. Speech came before parts-of-speech. Grammatically "ma" is as much of an interjection as "Fire!" But Whitney (53) says that interjections are not in the proper sense parts of speech.

When "E"' said “iah" (water) and è (eat), it was merely the outburst of an emotion. If analyzed it might be a halfdozen sentences. When she said "tê-tê" she wanted to say that there were wrinkles in her stomach which were making her uncomfortable. She had learned through experience that when she said "tē-tē" it meant pleasure. This is true of all early expressions. When " $E$ "' saw a dog, she saw an object in motion. Her parent said "dog," she imitating said dä. Mrs. Hall (10) states that "objects are seen as a whole without regard to either quality or motion." Motion is the first quality (?) of the object seen. It presents spatial qualities. It was there, now it is not there. This is what the child first sees. Motion rather than spatial relation or spatial qualities were observed when she said "dä." "Dä" was not an interjectional-substantive-attributive complex; it was a sentence-a thought expressed in words-no more, noless. When 
she said "doä" she meant that the door was in some way intimately connected with her desire. Either, here is a door; the door is shut; open the door, etc. "Doä" might convey or express all these meanings. The adult says "go" and expresses a sentence. The grammarian says this is elliptical, it must be expanded into a complete senterce, and then "go" is a verb. Unfortunately for both grammar and "E" she did not know this and called raisins "eats" and an alligator "bite." Parts of speech can only be classified according to the use of a word in a sentence.

Later more than one prominent word in the sentence is chosen. " $E$ "' in her second year said "opa doä." This is truly an abridged grammatical sentence. An imperative sentence. Negation was first expressed with the word "no" as a sort of enclytic. "Esbet go-no," meant: "Elizabeth does not want to go." "Yes" was not used until the 32 month. Before that time, "E"' would answer the question: Do you want this? with the word, "want." And the question: "Are you going?" with, "I are." "Are" came from pure imitation. Such solecisms result from the same cause and may be similarly classified with mispronunciation. The use and arrangement of words comes from imitation, the same as pronunciation. In some cases the child merely forgets. We say to the child; "You are," and he very naturally says "Yes, I are." Solecisms are always so plentiful and prominent in the speech of every child, that one is surprised that any one should ever have entertained the idea that if a child had never heard an incorrect grammatical expression he would never use one. Irregular inflections are very difficult for the child. Hence, " $E$ "' said, "He does n't know, do he?" "When did it flew away?" "You hurted my hand." The laws of pronunciation will take care of expressions like these: "I showed it at you." "Don't tell me any more questions."

Homer used the plural form before using the singular form, in noted instances. This is trie of the primitive man (Chamberlain (35)). " $E$ "' used many plural forms before using the singular. Shoes before shoe; toes before toe, teeth before tooth, etc. They are so listed in the Table of Vocabularies. The reason'for this is obvious-the child heard the plural form more frequently than the singular.

From a grammatical standpoint, " $E$ "' after using nouns and verbs, used adverbs, next came adjectives, pronouns, prepositions, and lastly conjunctions. Her first conjunction was "and" in her twentieth month. At two years of age she was using all the parts of speech and used simple, compound and complex sentences. 


\section{WORD CONTENT}

On "E's" fourth birthday the following words were given her to define. She was in each case asked: "What is - ?" "These "definitions" illustrate more associative memory than analysis and classification.

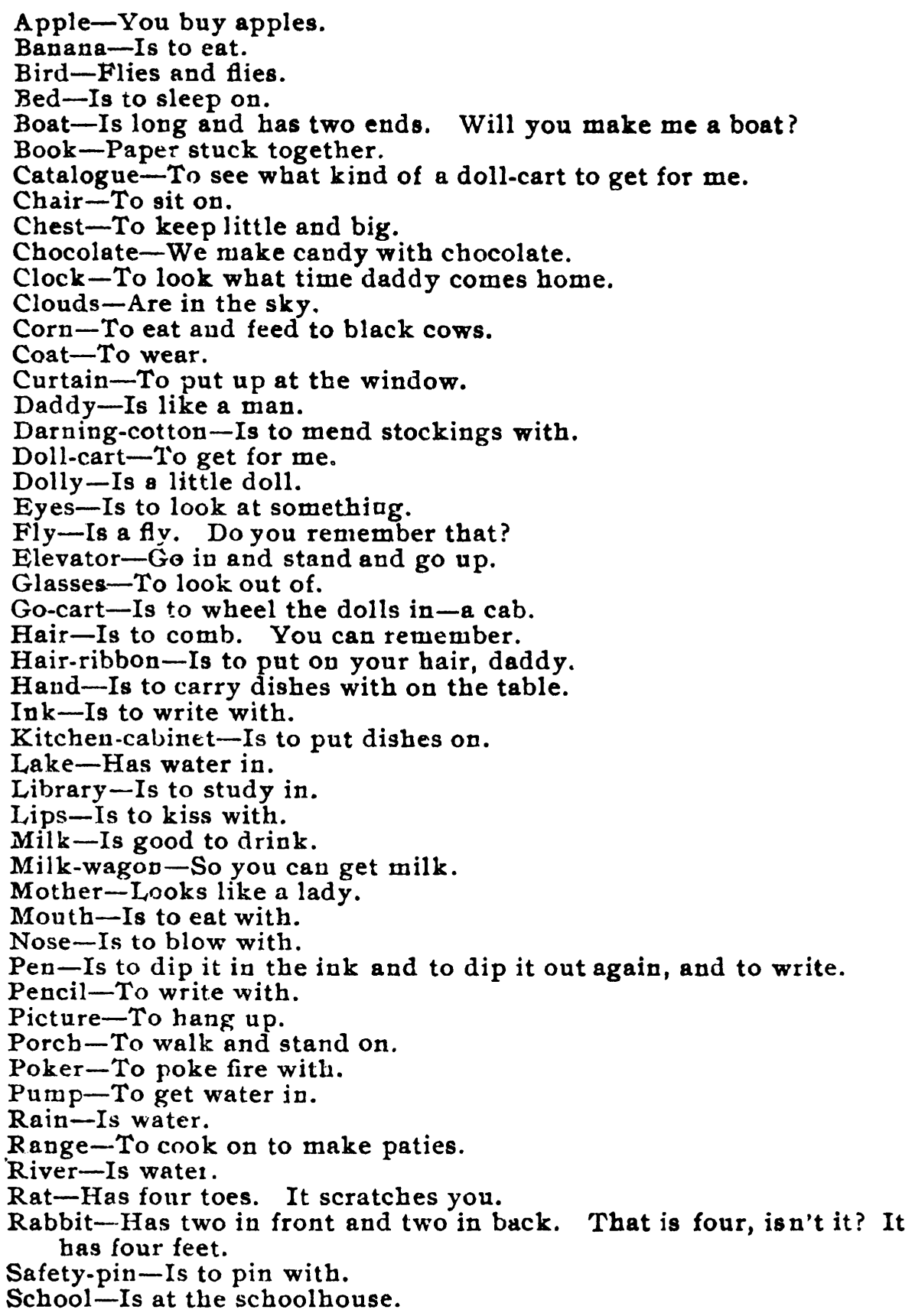


Shampoo-Put on your hair to get vasaline out.

Snow-Is white.

Stocking - To put on legs.

Stomach-Is to get full. I won't say any more.

Stove-To build fire with.

Strap-Is to paddle with.

Supporters-Is to fasten up stockings.

Switch-Is off trees.

Table-To eat on.

Teeth-Is to eat with.

Tea-Is to drink.

Tights-Is to keep warn.

Thread-Is to sew with.

Typewriter-To make letters.

Whip-Is to spank with.

Window-Is to look out of and to put up.

\section{CONCLUSION}

" $E$ "' is now nearly five years of age and uses approximately $\mathbf{r}, 800$ words. It is surprising to note how rapidly her vocabulary is increasing. She has attended kindergarten a few months and takes great interest in the work there.

Her color vocabulary has not increased except by a few terms for shades and tints. She can count to 1,000 and can tell the pages of a book up to $r, 000$. When a hymn is announced, as 187 , she very frequently can find it.

The writer intends to continue the enumeration of her vocabulary for succeeding years. It will soon be necessary to use different methods. By taking the unabridged dictionary, my own vocabulary two years ago was estimated at 44,000 by taking every 5oth page; and 43,000 by taking every Ioth page.

Correspondence in refefence to errors, or suggestions concerning this work, will be gladly received.

References Found Helpful. Magazines

I. Alimn, Margaret. "Notes on the Development of a Child's Language." (Mother's Nursery Guide, Feb., 1893.)

2. BARNES, E. "How Words Get Content." (Studies in Education, Vol. II, 43-6I.)

3. BuCkMaNN, S. S. "Speech of Children." (Nineteenth Century, Vol. XI,I, 793-807.)

4. Chamberlain, A. F. \& I. C. "Studies of a Child." (Ped.Sem.; Vol. XI, 264-29I.)

5. Conradi, E. "Speech Development of the Child." (Ped. Sem., Vol. XI, 328-380.)

6. DewEY, J. "The Psychology of Infant Language." (Psych. Rev., Vol. I, 63-66.)

7. GalE H. "The Vocabularies of Three Cbildren in One Family at Two and Three Years of Age." (Ped. Sem., Vol. IX, 422-435.)

8. Gala, H. "Recent Studies of Children's Vocabularies." (Child Study Mo., Vol. VI, 272-280.) 
9. GALE, C. M. \& H. "Children's Vocabularies." (Pop. Sci. Mo., Vol. LXI, 45-5I.)

10. Hal, W. W. (Mrs.). "First Five Hundred Days of a Child's Life." (Cuild Study Mo., Vol. II, 585-608.)

Ix. HOLDEN, E. $S$. "On the Vocabularies of Children under Two Years of Age." (Trans. Am. Phil. Assn., Vol. VIII, 58-68.)

12. HUMPHRFYS, W. "A Contribution to Infantile Linguistics." (Trans. Am. Phil. Assn., Vol. XII, 5-17.)

13. JEGI, J. "The Vocabulary of a Two-year-old Child." (Child Study Mo., Vol. VI, 24r-26r.)

14. KIRKPATRICK, E. A. "Number of Words in an Ordinary Vocabulary." (Science, Vol. XVIII, ro7-8.)

15. KIRKPATRICK, E A. "How Children Learn to Talk." (Science, Vol. XVIII, I75-6.)

16. KIRKPATkICK, E. A. "A Vocabulary Test." (Pop. Sci. Mo., Vol. LXX, 157-164.)

17. LuKENS, H. "Preliminary Report on the Learning of Language." (Ped. Sem., Vol. III, 424-460.)

18. MaCH, E. "Language: Its Origin, Development and Significance for Scientific Thought." (Open Court, Vol. XIV, I7I-I78.)

19. MATeER, Frorence. "The Vocabulary of a Four-year-old Boy." (Ped. Sem., Vol. XV, 63-74.)

20. MrckeNS, C. W. "Vocabulary." (Child Study Mo., Vol. III, 203-205.)

2I. MOORE, Kathrazs. "The Mental Development of a Child." (Psych. Rev., Mon. Sup., No. 3, 1886.)

22. Noblt, E. "Child Speech."' (Education, Vol. IX, 44-52.)

23. SAlisbury, A. "A Child's Vocabulary." (Educational Rev., Vol. VII, 289-293.;

24. SANFORD, E. C. "Notes on Studies of the Language of Children." (Ped. Sem., Vol. I, 257-260.)

25. Scriptura, E. W. "Terminal Verb in Infant Speech." (Science O. S. Vol., XXIII, 62.)

26. ShinN, Mili,icent. "Vocabularies." (Child Study Mo., Vol. VI, 398-40 (.)

27. Stavenson, A. "Speech of Children." (Science O. S., Vol. $\mathrm{XXI}, \mathrm{r}$ 18-120.)

28. Sorr, , J. "Baby Linguistics." (Eng. I11. Mag. Nov., 1884.)

29. TAINE, $H$. "Acquisition of Language by Children." (Mind, Vol. II, 252.)

30. TraCV, F. "The Language of Childhood." (Am. Jour. Psych., Vol. VI, 107-138.)

31. TRETIIHN, A. W. "The Psychology of Language Interest in Children." (Ped. Sem., Vol. XI, Ir 3-178.)

32. WhIPPLE, G. M. "Vocabulary and Word-building Test." (Psych. Rev., Vol. XV, 94-I05.)

33. WhIPPLE, G. M. "The Vocabulary of a Three-year-old Boy with some Interpretive Comments." (Ped. Sem., Vol. XVI, r-22.)

34. WorFE, H. K. "On the Color Vocabulary of Children." (Neb. University Studies, July, I890, 205-234.)

\section{RFFERENCES FOUND HELPFUI. BOOKS}

35. Chamberiain, A. F. "The Child: A Study in the Evolution of Man." London, 1903. (Chapter V.) 495 P.

36. Corrins, J. "The Faculty of Speech." New York, 1887. (Chapter III.) $430 \mathrm{P}$.

37. COMPaYRF, G. "Intellectual and Moral Development of a Child." New York, 18g6. (Chapter V.) 298 P. 


\section{A CHILD'S VOCABULARY 369}

38. JUDD, C. H. "Psychology: General Introduction." New York, 1907. $389 \mathrm{P}$.

39. King, I. "Psychology of Child Development." U. of C. Press, 1903. $265 \mathrm{P}$.

40. Kirkpatrick, E. A. "Fundamentals of Child Study." New York, $1907.384 \mathrm{P}$.

41. MeumanN, E. "Die Entstehung der Ersten Wortbedeutangen beim Kinde." Leipzig, 1908. I00 P.

42. MöLLER, MAX. "The Science of I,anguage." New York, 1862. (Ch. I.)

43. O'SheA, M. V. "Linguistic Development and Education." New York, 1907. (Chapter III.) 347 P.

44. PEREz, B. "The First Three Years of Childhood." London, 1889 .

45. Preyer, B. "The Senses and the Will." New York, I898. 353 P.

46. PREYHR, B. “The Derelopment of the Intellect." New York, 1889. $317 \mathrm{P}$.

47. Romanes, C. J. "Mental Erolution in Man." New York, I889. (Chapter VI.) $452 \mathrm{P}$.

48. Schul, Tz, F. "Die Sprache des Kindes." Leipzig, I88o. 46 P.

49. Shinn, Millicent. "Biography of a Baby." New York, 1900. $247 \mathrm{P}$.

50. Sul,y, J. "Studies of Childhood." New York, 1898. 527 P.

5I. TRACY, F. "The Psychology of Childhood." Boston, 1895. $170 \mathrm{P}$.

52. TANNeR, Amy E. "The Child." Chicago, 1904. $430 \mathrm{P}$.

53. WhirneY, W. P. "Language." New York, 1887. (Chapter XI.)

54. WUNDT, W. "Outline of Psychology." (Trans. Dr. C. H. Judd, New York, 1897.) (Especially Chapter IV.) 342 P.

55. WundT, W. "Völker-Psychologie." Leipzig, rgoo. (Pt. I, 627644.) 644 P. 